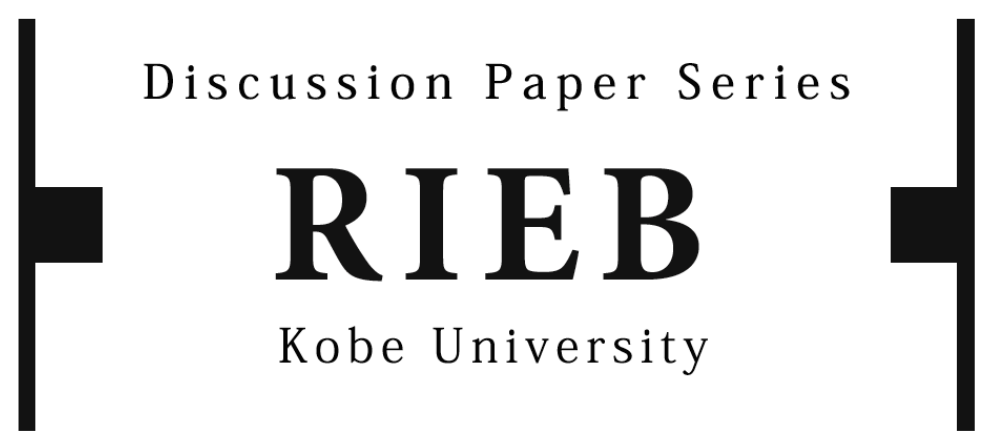

DP2018-23

\title{
The Pivotal Mechanism Versus the Voluntary Contribution Mechanism: An Experimental Comparison
}

Tatsuki HOMMA Junyi SHEN

Takuma WAKAYAMA Hirofumi YAMAMURA

Takehiko YAMATO

November 5, 2018

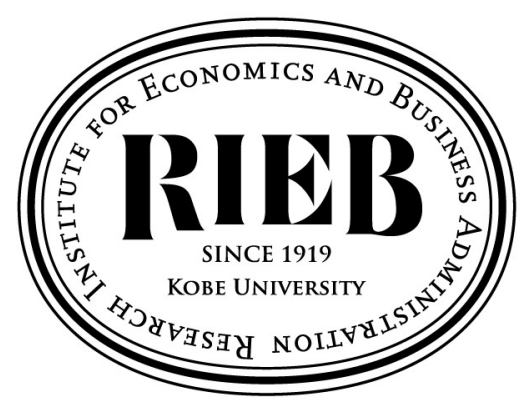

Research Institute for Economics and Business Administration Kobe University 


\title{
The pivotal mechanism versus the voluntary contribution mechanism: An experimental comparison*
}

\author{
Tatsuki Homma $^{\dagger}$ Junyi Shen ${ }^{\ddagger}$ Takuma Wakayama ${ }^{\S}$ \\ Hirofumi Yamamura Takehiko Yamatoll
}

November 5, 2018

\begin{abstract}
We conduct an experimental comparison of two well-known mechanisms for undertaking a binary public project: the pivotal mechanism and the voluntary contribution mechanism. We compare the two mechanisms under complete information in which each subject knows the other subjects' payoffs. We then observe that the voluntary contribution mechanism works better than the pivotal mechanism from the perspectives of Pareto efficiency and individual rationality. On the other hand, we observe that the pivotal mechanism works better than the voluntary contribution mechanism in light of decision efficiency. These results suggest that whenever we insist on either Pareto efficiency or individual rationality, the voluntary contribution mechanism is more favorable than the pivotal mechanism in practical applications.
\end{abstract}

Keywords: laboratory experiment; pivotal mechanism; voluntary contribution mechanism

JEL codes: C92; D71; D78; H41

*This work was supported by JSPS KAKENHI Grant Numbers JP22730165, JP22330061, JP25380244, JP26285045, JP15H03328, and JP16K03567.

$\dagger$ Department of Social Engineering, Graduate School of Decision Science and Technology, Tokyo Institute of Technology. E-mail: homma.t.aa@gmail.com

$\ddagger$ Research Institute for Economics and Business Administration, Kobe University. E-mail: shen@rieb.kobe-u.ac.jp

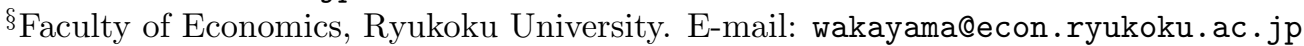

"Department of Law and Economics, School of Economics, Hokusei Gakuen University. E-mail: h-yamamura@hokusei.ac.jp

"Department of Industrial Engineering and Economics, School of Engineering, Tokyo Institute of Technology. E-mail: yamato.t.aa@m.titech.ac.jp 


\section{Introduction}

We consider an economy with a public good in which agents are facing a decision of whether to undertake a project that produces a fixed level of the public good. The public project should be undertaken if the sum of agents' true values of the project is larger than or equal to the cost of undertaking the project, but not otherwise. However, this condition of decision efficiency is difficult to satisfy since the mechanism designer does not know each agent's true value of the public project, and the agent may have an incentive to "free-ride" payments from other agents and undertake the project while lying about their own values.

There are two well-known mechanisms to solve this free-rider problem theoretically: the pivotal mechanism and the voluntary contribution (or provision point) mechanism. In the pivotal mechanism due to Clark (1971), reporting their true value of the project is a dominant strategy for everyone. Therefore, this mechanism satisfies the decision efficiency condition. However, the rule of the mechanism is complicated and dominant strategy equilibrium allocations of the mechanism may be neither Pareto efficient nor individually rational.

On the other hand, the rule of the voluntary contribution mechanism is simple, and the mechanism implements the core when the equilibrium concept is undominated perfect equilibrium, which is a refinement of Nash equilibrium (Bagnoli and Lipman, 1989). Then, all equilibrium allocations of the mechanism are Pareto efficient and individually rational. The mechanism also satisfies decision efficiency. We should however note that this equilibrium concept requires complete information among agents: everyone knows the payoffs of all other agents.

The above theoretical predictions suggest that the voluntary contribution mechanism would perform better than the pivotal mechanism under complete information. This motivates us to investigate whether these predictions are correct in a laboratory experiment. Indeed, several papers have examined how one of the two mechanisms works in laboratory experiments. ${ }^{1}$ However, these experimental studies cannot specify which of these two mechanisms is better because they focused either on the pivotal mechanism or on the voluntary contribution mechanism. To the best of our knowledge, no paper experimentally compares the two mechanisms in the same economic environment with a public project.

The purpose of this paper is to investigate which mechanism, the pivotal mechanism or the voluntary contribution mechanism, should be used in the provision of

\footnotetext{
${ }^{1}$ See Chen (2008) and Chen and Ledyard (2010) for excellent surveys on these experimental results.
} 
a binary public project by conducting an experimental comparison. In designing an experiment to compare the two mechanisms, we pay close attention to the following two points. First, the comparison should be made under the same economic environmental conditions on the number of subjects, the true values that subjects receive from the project, the cost of undertaking the project, and the informational condition. We compare the two mechanisms under complete information in which each subject knows the true values of all subjects.

The second point we are concerned with in comparing the two mechanisms is to hold the complexity constant. Clearly, the rule of the pivotal mechanism is more complicated than that of the voluntary contribution mechanism. Attiyeh et al. (2000) and Kawagoe and Mori (2001) argued that subjects suffered from confusion due to the complexity of the pivotal mechanism and the non-transparency of the dominant strategy. To eliminate such confusion, following Cason et al. (2006), we focused on the two-agent case and simply presented to each subject a payoff table describing the relation between outcomes and choices by the subject and the others without explaining the rule of the pivotal mechanism. Similarly, for the voluntary contribution mechanism experiment, we used payoff tables only and gave no explanation on the rule of the mechanism. The use of payoff tables allows for a comparison of the pivotal mechanism with the voluntary contribution mechanism holding the degree of transparency constant.

We compare the two mechanisms from four different normative criteria: the Pareto efficiency index, the individual rationality index, the decision efficiency index, and the surplus index. Here, the Pareto efficiency index is the ratio of Pareto efficient outcomes. The individual rationality index is the ratio of individually rational outcomes in which each subject receives a payoff at least as large as their own before participating in the mechanism, that is, the payoff the subject receives from the initial endowment of a private good without any public project. The decision efficiency index is the ratio of decision-efficient outcomes in which the public project is undertaken because the sum of the subjects' true values of the project exceeds the cost of undertaking the project in our experimental setting. The surplus index is a measure of the gains subjects obtain by participating in a mechanism, which is a percentage of the highest possible payoff that could be achieved in the mechanism.

We observed that the Pareto efficiency index, the individual rationality index, and the surplus index for the voluntary contribution mechanism were significantly higher than those for the pivotal mechanism. On the other hand, the decision efficiency index for the voluntary contribution mechanism was significantly lower than that for the pivotal mechanism. These results suggest that whenever we insist on either Pareto 
efficiency or individual rationality, the voluntary contribution mechanism is more favorable than the pivotal mechanism in practical applications.

The remainder of the paper is organized as follows. Section 2 presents a brief review of the laboratory evidence on the pivotal and voluntary contribution mechanisms. In Section 3, we describe the model of a public project and the definitions of mechanisms. We also propose a new equilibrium concept and provide theoretical prediction results that are useful in examining our experimental observations. Section 4 describes our experimental design. We explain the experimental results in Section 5. Section 6 provides concluding remarks. The appendix contains the proofs of our theoretical results and our experimental instructions.

\section{Related literature}

Attiyeh et al. (2000) conducted laboratory experiments on the pivotal mechanism and found that few subjects reported their true values for the public project when only the rule of the pivotal mechanism was explained to groups of five or ten subjects. Less than $10 \%$ of the bids were truth-telling in incomplete information settings in which each subject knew their own true value but not the others' values in the same group. Kawagoe and Mori (2001) also found that the ratio of the truth-telling bids significantly increased from $16 \%$ to $47 \%$ when payoff tables were given to subjects in addition to an explanation of the rule of the pivotal mechanism. Moreover, Cason et al. (2006) experimentally compared the Groves mechanism, that is "secure" in the sense that the set of dominant strategy equilibrium outcomes coincides with the set of Nash equilibrium outcomes, with the pivotal mechanism that is not secure. They observed that the ratio of dominant strategies that subjects chose was significantly higher in the secure Groves mechanism than in the non-secure pivotal mechanism.

For the voluntary contribution mechanism with a single unit of public good, laboratory experimental studies found limited support for the theoretical prediction that efficient outcomes are achieved. For example, Marks and Croson (1999) observed that the ratio that decision efficiency was satisfied was $48 \%$ under complete information and $54 \%$ under incomplete information, and that the ratio of Pareto efficient equilibrium strategies subjects played was $4 \%$ under complete information and $6 \%$ under incomplete information, even though group contributions converged toward the equilibrium level over time both under complete information and under incomplete information. There are other laboratory experimental studies examining the effects of changing details of the voluntary contribution mechanism such as refund rules, rebate rules, incomplete in- 
formation on the number of players or the cost of the public project, and simultaneous versus sequential contributions (see Chen (2008) for a survey).

Healy (2006) conducted an experimental comparison of five public good mechanisms, the voluntary contribution, proportional taxation, Groves-Ledyard, Walker, and Vickrey-Clarke-Groves (VCG) mechanisms, in the same economic environment with five-person groups under incomplete information. He found that the VCG mechanism was the most efficient among the five mechanisms. In his experimental setting, the level of the public good is chosen from a continuum, and each subject knows their own preference parameters but not the preference parameters of others. On the other hand, the public project choice is binary in our experiment, and we investigate the complete information case. Moreover, we compare the voluntary contribution mechanism with the pivotal mechanism which is the VCG mechanism with a binary public project from the viewpoints of decision efficiency and individual rationality as well as Pareto efficiency.

\section{Preliminaries}

\subsection{Model}

We consider a two-agent economy with a binary public project. Two agents face a decision whether to undertake a public project. Let $y \in\{0,1\}$ be a public project. In our model, $y=1$ if the public project is undertaken, and $y=0$ otherwise. The cost of undertaking a public project is $c>0$. Let $t=\left(t_{1}, t_{2}\right) \in \mathbb{R}^{2}$ be a transfer vector, where $t_{i}$ denotes the payment for agent $i$. We denote the set of feasible allocations by

$$
A=\left\{(y, t) \in\{0,1\} \times \mathbb{R}^{2}:-\left(t_{1}+t_{2}\right) \geq c \cdot y\right\}
$$

For each $i \in\{1,2\}$, let

$$
A_{i}=\left\{\left(y, t_{i}\right) \in\{0,1\} \times \mathbb{R}:-t_{i} \geq c \cdot y\right\}
$$

be the set of $i$-feasible allocations. Let $\omega_{i}>0$ be agent $i$ 's initial endowment.

Each agent $i \in\{1,2\}$ has a quasi-linear preference relation over $\{0,1\} \times \mathbb{R}$, which is described by the value of the public project $v_{i} \geq 0$. Denote the set of such values of agent $i$ by $V_{i}$. That is, given any $i \in\{1,2\}$ and any $v_{i} \in V_{i}$, $i$ 's preference relation is represented by the function $u_{i}\left(\cdot, \cdot ; v_{i}\right):\{0,1\} \times \mathbb{R} \rightarrow \mathbb{R}$ defined for each $\left(y, t_{i}\right) \in$ 
$\{0,1\} \times \mathbb{R}$, by

$$
u_{i}\left(y, t_{i} ; v_{i}\right)=v_{i} \cdot y+\omega_{i}+t_{i}
$$

Let $v=\left(v_{1}, v_{2}\right) \in V \equiv V_{1} \times V_{2}$ be a value profile.

\subsection{Properties of allocations}

We introduce some standard properties of allocations. An allocation $(y, t) \in A$ is Pareto efficient for $v \in V$ if there is no allocation $\left(y^{\prime}, t^{\prime}\right) \in A$ such that $u_{i}\left(y^{\prime}, t_{i}^{\prime} ; v_{i}\right) \geq u_{i}\left(y, t_{i} ; v_{i}\right)$ for each $i \in\{1,2\}$ with a strict inequality for some $j \in\{1,2\}$. We denote the set of Pareto efficient allocations for $v$ by $P(v)$. The following fact characterizes the set of Pareto efficient allocations.

Fact 1. Let $v \in V$. An allocation $(y, t) \in A$ is Pareto efficient for $v$ if and only if

$$
u_{1}\left(y, t_{1} ; v_{1}\right)+u_{2}\left(y, t_{2} ; v_{2}\right)= \begin{cases}v_{1}+v_{2}-c+\omega_{1}+\omega_{2} & \text { if } v_{1}+v_{2} \geq c \\ \omega_{1}+\omega_{2} & \text { otherwise }\end{cases}
$$

An allocation $(y, t) \in A$ is decision efficient for $v \in V$ if $y \in \arg \max _{y^{\prime} \in\{0,1\}}\left(v_{1}+\right.$ $\left.v_{2}\right) \cdot y^{\prime}-c \cdot y^{\prime}$. The following fact states that decision efficiency is weaker than Pareto efficiency.

Fact 2. Let $v \in V$. If an allocation $(y, t) \in A$ is Pareto efficient for $v$, then it is decision efficient for $v$.

An allocation $(y, t) \in A$ is individually rational for $v \in V$ if $u_{i}\left(y, t_{i} ; v_{i}\right) \geq u_{i}\left(y^{\prime}, t_{i}^{\prime} ; v_{i}\right)$ for each $i \in\{1,2\}$ and each $\left(y^{\prime}, t_{i}^{\prime}\right) \in A_{i}$. We denote the set of individually rational allocations for $v$ by $I(v)$. The following fact characterizes the set of individually rational allocations.

Fact 3. Let $v \in V$. An allocation $(y, t) \in A$ is individually rational for $v$ if and only if for each $i \in\{1,2\}$,

$$
u_{i}\left(y, t_{i} ; v_{i}\right) \geq \begin{cases}\omega_{i} & \text { if } c>v_{i} \\ v_{i}-c+\omega_{i} & \text { otherwise. }\end{cases}
$$

The core for $v \in V$ is the set of allocations that are Pareto efficient and individually 
rational for $v .^{2}$ We denote the core for $v$ by $C(v)$. That is, $C(v)=P(v) \cap I(v)$ for each $v \in V$.

\subsection{Equilibrium concepts}

A mechanism is a pair $\Gamma=(S, g)$, where $S \equiv S_{1} \times S_{2}, S_{i}$ is strategy space of agent $i$, and $g: S \rightarrow A$ is an outcome function. Given $v \in V$, a pair $(\Gamma, v)$ constitutes a (normal form) game.

We now introduce several equilibrium concepts.

- Dominant strategy equilibrium. A strategy profile $\left(s_{1}, s_{2}\right) \in S$ is a dominant strategy equilibrium of the game $(\Gamma, v)$ if

(i) for each $s_{1}^{\prime} \in S_{1}$ and each $s_{2}^{\prime} \in S_{2}, u_{1}\left(g_{1}\left(s_{1}, s_{2}^{\prime}\right) ; v_{1}\right) \geq u_{1}\left(g_{1}\left(s_{1}^{\prime}, s_{2}^{\prime}\right) ; v_{1}\right)$; and

(ii) for each $s_{1}^{\prime} \in S_{1}$ and each $s_{2}^{\prime} \in S_{2}, u_{2}\left(g_{2}\left(s_{1}^{\prime}, s_{2}\right) ; v_{2}\right) \geq u_{2}\left(g_{2}\left(s_{1}^{\prime}, s_{2}^{\prime}\right) ; v_{2}\right)$.

- Nash equilibrium. A strategy profile $\left(s_{1}, s_{2}\right) \in S$ is a Nash equilibrium of the game $(\Gamma, v)$ if

(i) for each $s_{1}^{\prime} \in S_{1}, u_{1}\left(g_{1}\left(s_{1}, s_{2}\right) ; v_{1}\right) \geq u_{1}\left(g_{1}\left(s_{1}^{\prime}, s_{2}\right) ; v_{1}\right)$; and

(ii) for each $s_{2}^{\prime} \in S_{2}, u_{2}\left(g_{2}\left(s_{1}, s_{2}\right) ; v_{2}\right) \geq u_{2}\left(g_{2}\left(s_{1}, s_{2}^{\prime}\right) ; v_{2}\right)$.

- Undominated Nash equilibrium. Given any $i \in\{1,2\}$, we say that an agent $i$ 's strategy $s_{i} \in S_{i}$ is weakly dominated in the game $(\Gamma, v)$ if there is $s_{i}^{\prime} \in S_{i}$ such that for each $s_{j} \in S_{j}, u_{i}\left(g_{i}\left(s_{i}^{\prime}, s_{j}\right) ; v_{i}\right) \geq u_{i}\left(g_{i}(s) ; v_{i}\right)$ with a strict inequality for some $s_{j}^{\prime} \in S_{j}$. Denote the set of $i$ 's strategies that are not weakly dominated in the game $(\Gamma, v)$ by $U\left(S_{i} ;(\Gamma, v)\right)$. A strategy profile $\left(s_{1}, s_{2}\right) \in S$ is an undominated Nash equilibrium of the game $(\Gamma, v)$ if (i) it is a Nash equilibrium of $(\Gamma, v)$ and (ii) $s_{i} \in U\left(S_{i} ;(\Gamma, v)\right)$ for each $i \in\{1,2\}$.

- Twice iteratively undominated Nash equilibrium. Let $U(S ;(\Gamma, v)) \equiv$ $U\left(S_{1} ;(\Gamma, v)\right) \times U\left(S_{2} ;(\Gamma, v)\right)$ and $\Gamma(U) \equiv(U(S ;(\Gamma, v)), g)$. A strategy profile $\left(s_{1}, s_{2}\right)$ $\in S$ is a twice iteratively undominated Nash equilibrium of the game $(\Gamma, v)$ if it is an undominated Nash equilibrium of the game $(\Gamma(U), v)$. Let $\mathcal{E}(\Gamma, v)$ and $\mathcal{A}(\Gamma, v)$ be the set of twice iteratively undominated Nash equilibria for $(\Gamma, v)$ and the set of twice iteratively undominated Nash equilibrium allocations for $(\Gamma, v)$, respectively.

\footnotetext{
${ }^{2}$ This definition depends on the number of agents. When there are three or more agents, the core is generally smaller than the set of allocations satisfying Pareto efficiency and individual rationality.
} 


\subsection{Voluntary contribution mechanism}

We consider two mechanisms that play central roles in the literature. Our first mechanism is the following simple mechanism that is widely used in practice: each agent offers a voluntary contribution. If the sum of the contributions covers the cost of the public project, it is undertaken; otherwise, the contributions are returned. Formally:

The voluntary contribution mechanism, $\Gamma^{\mathbf{V C}}=\left(\boldsymbol{S}^{\mathbf{V C}}, \boldsymbol{g}^{\mathbf{V C}}\right)$. For each $i \in\{1,2\}$, $S_{i}^{\mathrm{VC}}=\left[0, \omega_{i}\right]$; and for each $s \in S^{\mathrm{VC}}$,

$$
g^{\mathrm{VC}}(s)=\left(y^{\mathrm{VC}}(s),\left(t_{1}^{\mathrm{VC}}(s), t_{2}^{\mathrm{VC}}(s)\right)\right)= \begin{cases}(0,(0,0)) & \text { if } s_{1}+s_{2}<c \\ \left(1,\left(-s_{1},-s_{2}\right)\right) & \text { otherwise }\end{cases}
$$

This subsection now makes the following assumption:

A1. For each $v \in V$ and each $i \in\{1,2\}, \omega_{i} \geq c>v_{i}$.

A1 says that each agent's initial endowment exceeds the cost of undertaking a public project, and each agent's value is less than the cost of undertaking a public project. A1 also says that no agent would like to contribute more than their initial endowment. ${ }^{3}$ We conduct laboratory experiments in the environments that satisfy A1.

Under A1, Facts 1 and 3 together imply that for each $v \in V$,

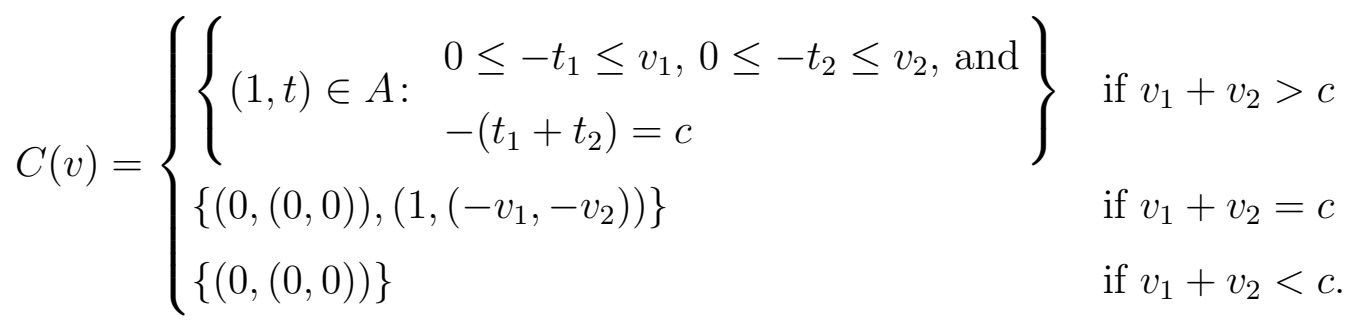

The next proposition states that the twice iteratively undominated Nash equilibrium allocations of the voluntary contribution mechanism are in the core for true preferences. $^{4}$

\footnotetext{
${ }^{3}$ Instead of A1, Bagnoli and Lipman (1989) used the following weaker assumption: $\omega_{1}+\omega_{2}>c$ and for each $v \in V$ and each $i \in\{1,2\}, \omega_{i}>v_{i}$.

${ }^{4}$ This is equivalent to stating that the voluntary contribution mechanism implements a subcorrespondence of the core in twice iteratively undominated Nash equilibria. Moreover, we could argue that the voluntary contribution mechanism "almost" fully implements the core in twice iteratively undominated Nash equilibria because the following facts hold: for each $v \in V$, (i) if $v_{1}+v_{2}>c$, then $C(v) \backslash \mathcal{A}\left(\Gamma^{\mathrm{VC}}, v\right)=\left\{\left(1,\left(-v_{1}, v_{1}-c\right)\right),\left(1,\left(v_{2}-c,-v_{2}\right)\right)\right\}$; (ii) if $v_{1}+v_{2}=c$, then $C(v) \backslash \mathcal{A}\left(\Gamma^{\mathrm{VC}}, v\right)=\left\{\left(1,\left(-v_{1},-v_{2}\right)\right)\right\}$; and (iii) if $v_{1}+v_{2}<c$, then $C(v)=\mathcal{A}\left(\Gamma^{\mathrm{VC}}, v\right)$.
} 
Proposition 1. Assume A1. Let $v \in V$. Then,

$$
\mathcal{A}\left(\Gamma^{\mathrm{VC}}, v\right) \subseteq C(v)
$$

The voluntary contribution mechanism is simple and, thus, it can be easily implemented in practice. However, to obtain allocations that are in the core via the voluntary contribution mechanism, agents are required to have a higher degree of rationality, and the mechanism designer must understand that a value profile is common knowledge between agents.

Remark 1. Our notion of twice iteratively undominated Nash equilibrium is different from the notion of iterated elimination of weakly dominated strategies. The reason we do not employ the notion of iterated elimination of weakly dominated strategies is that the voluntary contribution mechanism cannot implement the core (and any sub-correspondence of the core) in iterated elimination of weakly dominated strategies; that is, in the voluntary contribution mechanism, some allocations obtained by iterated elimination of weakly dominated strategies do not belong to the core. See Appendix B for a more detailed discussion.

Remark 2. To show that the voluntary contribution mechanism implements the core, Bagnoli and Lipman (1989) used another refinement of Nash equilibrium called "undominated perfect equilibrium" that applies the notion of (trembling-hand) perfection to the game after eliminating dominated strategies of the original game. However, they did not directly apply the notion of perfection to the infinite game associated with the voluntary contribution mechanism. ${ }^{5}$ Instead, they defined an undominated perfect equilibrium in the infinite game associated with the voluntary contribution mechanism as the limit of some sequence of undominated perfect equilibria of approximating finite versions of the voluntary contribution mechanism. Thus, it is an interesting open question whether a similar implementation result can be obtained when perfection is directly defined on the infinite game associated with the voluntary contribution mechanism.

\footnotetext{
${ }^{5}$ Recently, Carbonell-Nicolau (2011) provided a notion of perfection in infinite games. By invoking his notion of perfection, he examined the existence of undominated perfect equilibrium in the infinite game associated with the voluntary contribution mechanism. However, it is an open question whether a similar implementation result holds for his notion of perfection.
} 


\subsection{Pivotal mechanism}

Our second mechanism determines the allocation as follows: each agent $i \in\{1,2\}$ reports their value $v_{i}$ of the public project simultaneously. If $v_{1}+v_{2} \geq c$, then the public project is undertaken, and it is not undertaken otherwise. If the public project is undertaken, then both agents pay for an equal share of the project's cost. Moreover, whether the public project is undertaken or not, each agent $i \in\{1,2\}$ must pay the pivotal tax $p_{i}$ defined as follows:

$$
p_{i}= \begin{cases}-\left(v_{j}-\frac{c}{2}\right) & \text { if } v_{1}+v_{2} \geq c \text { and } v_{j}-\frac{c}{2}<0 \\ v_{j}-\frac{c}{2} & \text { if } v_{1}+v_{2}<c \text { and } v_{j}-\frac{c}{2}>0 \\ 0 & \text { otherwise. }\end{cases}
$$

The pivotal tax is the actual net benefit the other agent enjoys minus the maximal net benefit the agent can enjoy under the equal share cost. Thus, the pivotal tax is the social cost of considering agent $i$ 's preference. Formally:

The pivotal mechanism, $\boldsymbol{\Gamma}^{\mathbf{P}}=\left(\boldsymbol{S}^{\mathbf{P}}, \boldsymbol{g}^{\mathbf{P}}\right)$. For each $i \in\{1,2\}, S_{i}^{\mathrm{P}}=V_{i}$; and for each $v \in V=S^{\mathrm{P}}$, we denote $g^{\mathrm{P}}(v)=\left(y^{\mathrm{P}}(v),\left(t_{1}^{\mathrm{P}}(v), t_{2}^{\mathrm{P}}(v)\right)\right)$, where

$$
\begin{aligned}
& y^{\mathrm{P}}(v) \in \arg \max _{y \in\{0,1\}}\left(v_{1}+v_{2}\right) \cdot y+c \cdot y, \\
& t_{1}^{\mathrm{P}}(v)=-\frac{c \cdot y^{\mathrm{P}}(v)}{2}+\left(v_{2} \cdot y^{\mathrm{P}}(v)-\frac{c \cdot y^{\mathrm{P}}(v)}{2}\right)-\max _{y \in\{0,1\}}\left(\frac{c \cdot y}{2}+v_{2} \cdot y\right), \\
& t_{2}^{\mathrm{P}}(v)=-\frac{c \cdot y^{\mathrm{P}}(v)}{2}+\left(v_{1} \cdot y^{\mathrm{P}}(v)-\frac{c \cdot y^{\mathrm{P}}(v)}{2}\right)-\max _{y \in\{0,1\}}\left(\frac{c \cdot y}{2}+v_{1} \cdot y\right) .
\end{aligned}
$$

The pivotal mechanism might appear less simple than the voluntary contribution mechanism. However, it is well known that under the pivotal mechanism, truth-telling is a dominant strategy for everyone. Therefore, in contrast to the voluntary contribution mechanism, in the pivotal mechanism, agents need not care about the exact value of the other agent when considering their own action. Moreover, although the dominant strategy equilibrium allocations of this mechanism may not be Pareto efficient or individually rational, they are always decision efficient. 


\section{Experimental design}

Our experiment studies the voluntary contribution mechanism and the pivotal mechanism under complete information. Treatment V implements the voluntary contribution mechanism for a two-agent group. Treatment $\mathrm{P}$ implements the pivotal mechanism for a two-agent group. The public project problem to be solved is the same for both treatments. There are two types of agents, 1 and 2. The initial endowment vector of the private good is given by $\left(\omega_{1}, \omega_{2}\right)=(20,20)$. The true value profile $\left(v_{1}, v_{2}\right)$ is equal to $(9,13)$. The cost of undertaking the public project is $c=20$. By decision efficiency introduced in Section 3.2, the public project should be undertaken since $v_{1}+v_{2}-c=2>0$. Note that this environment satisfies A1 mentioned in Section 3.4.

\subsection{Treatment V}

Let the strategy space of each type be the set of integers from 0 to 20 . According to the rules of the voluntary contribution mechanism explained in Section 3.4, we can construct the payoff matrices of Types 1 and 2. The payoff tables that we employ in Treatment $\mathrm{V}$ are Tables 1 and 2 whose structures are the same as the original payoff tables with the exception that a linear transformation of the valuation functions is employed: $10 u_{i}-40$ for each type $i \in\{1,2\}$.

Table 1 (resp. Table 2) is a payoff table of Type 1 (resp. Type 2) with both types' payoffs displayed: the lower left-hand number is Type 1's (resp. Type 2's) payoff, and the upper right-hand number is Type 2's (resp. Type 1's) payoff in each cell. Table 1 also specifies the Nash equilibria and the unique twice iteratively undominated Nash equilibrium. ${ }^{6}$ In Table 1 , there are three "good" Nash equilibria, $(7,13),(8,12)$, and $(9,11)$, in the sense that the public project is undertaken and, hence, decision efficiency is met, whereas there are ninety-six "bad" Nash equilibria in the sense that the public project is not undertaken and, thus, decision efficiency is not satisfied. ${ }^{7}$ Among these Nash equilibria, only the pair $\left(s_{1}, s_{2}\right)=(8,12)$ is a twice iteratively undominated Nash equilibrium. Note that each Type 1 's strategy $s_{1} \in\{9, \ldots, 20\}$ is weakly dominated by $s_{1}=8$, whereas each strategy $s_{1} \in\{0, \ldots, 7\}$ is not weakly dominated by any strategy. Similarly, each Type 2's strategy $s_{2} \in\{13, \ldots, 20\}$ is weakly dominated by $s_{2}=12$, whereas each strategy $s_{2} \in\{0, \ldots, 11\}$ is an undominated strategy.

Eliminating these weakly dominated strategies of two players from Table 1 of the

\footnotetext{
${ }^{6}$ In the payoff table that we distributed to subjects, there was no tag and highlighting indicating the equilibria as in Table 1.

${ }^{7}$ The set of "bad" Nash equilibria is $\left\{\left(s_{1}, s_{2}\right) \in S: s_{1} \in\{0, \ldots, 7\}\right.$ and $\left.s_{2} \in\{0, \ldots, 11\}\right\}$.
} 


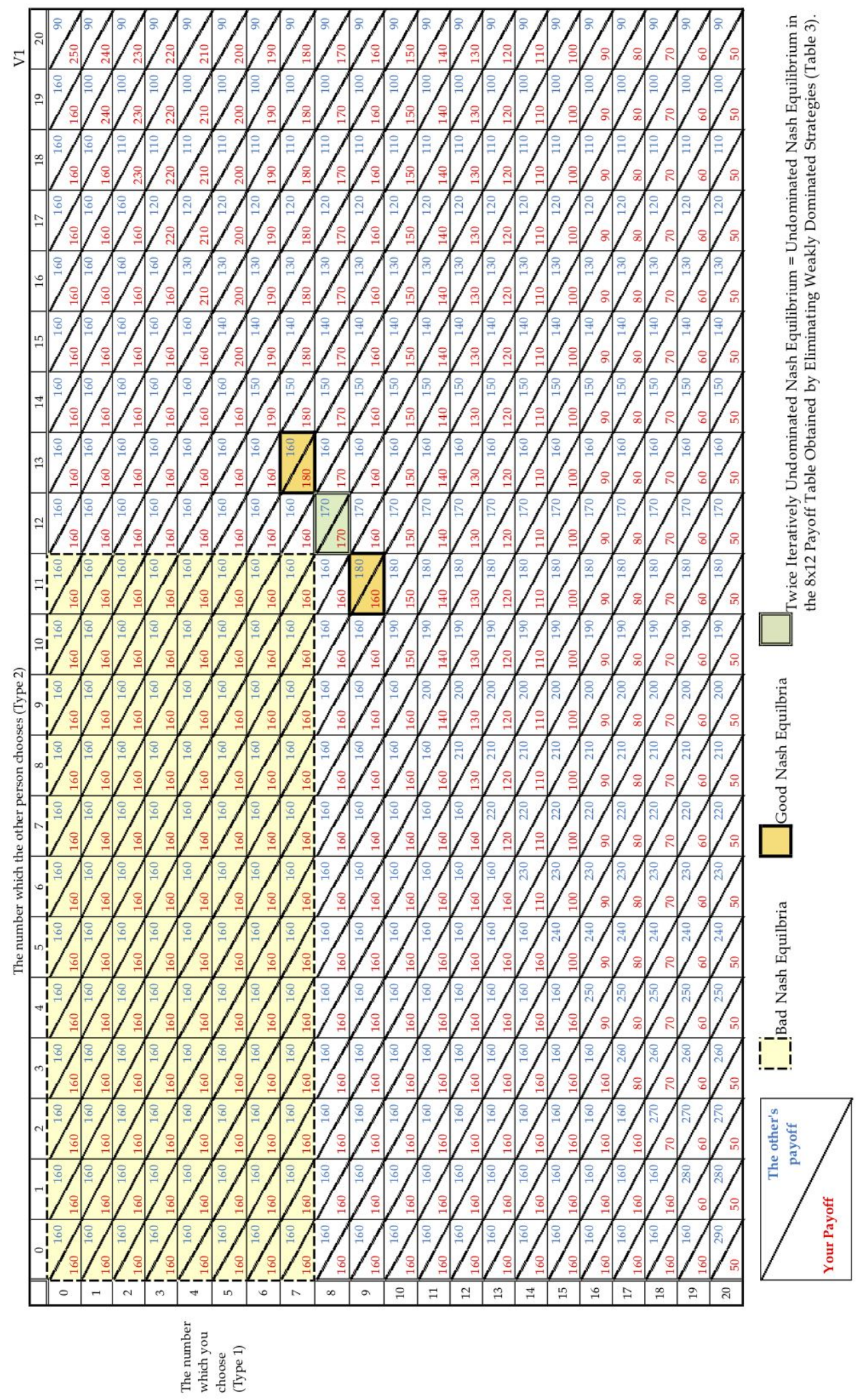

Table 1: Payoff table of Type 1 in Treatment V. 


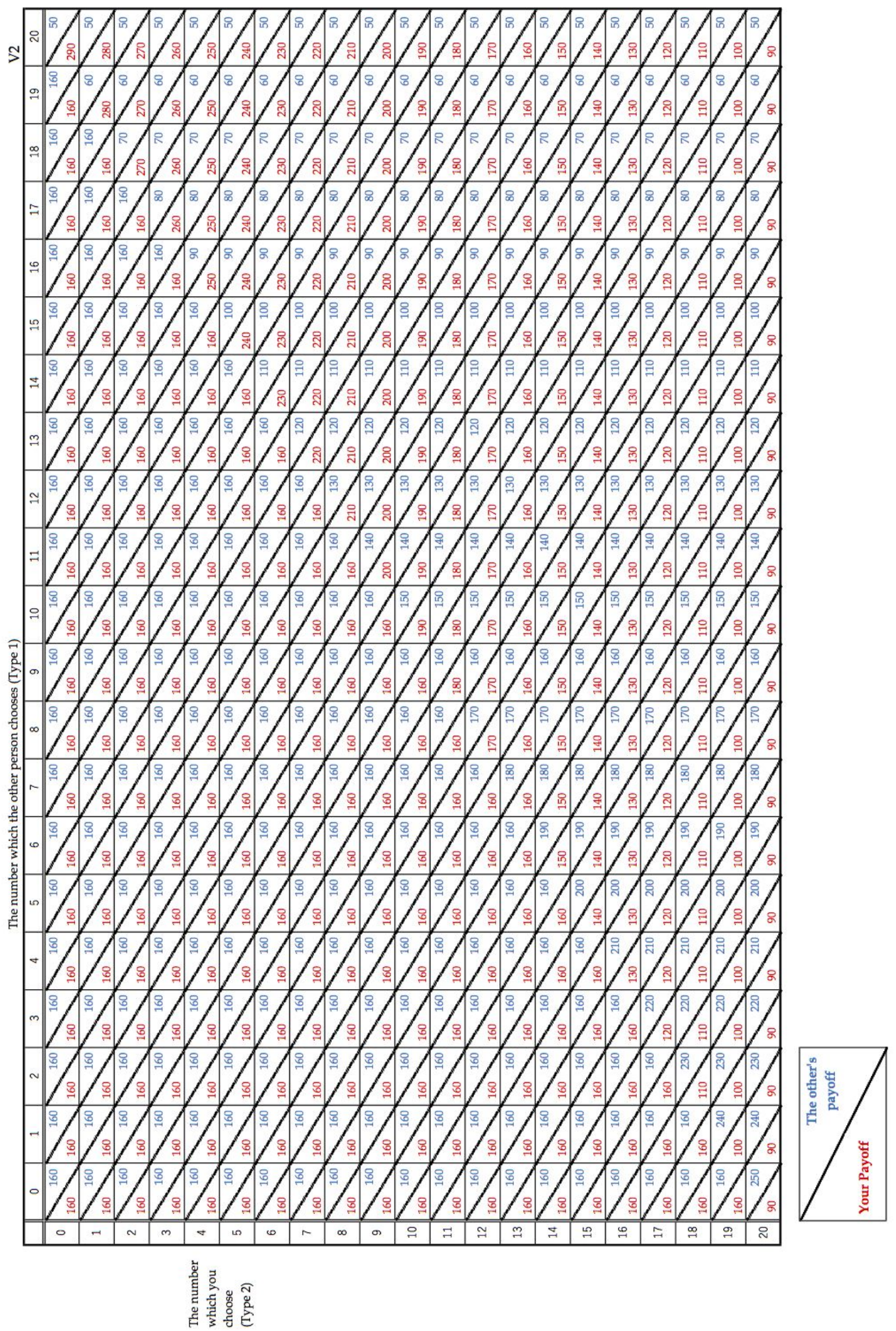

Table 2: Payoff table of Type 2 in Treatment V. 


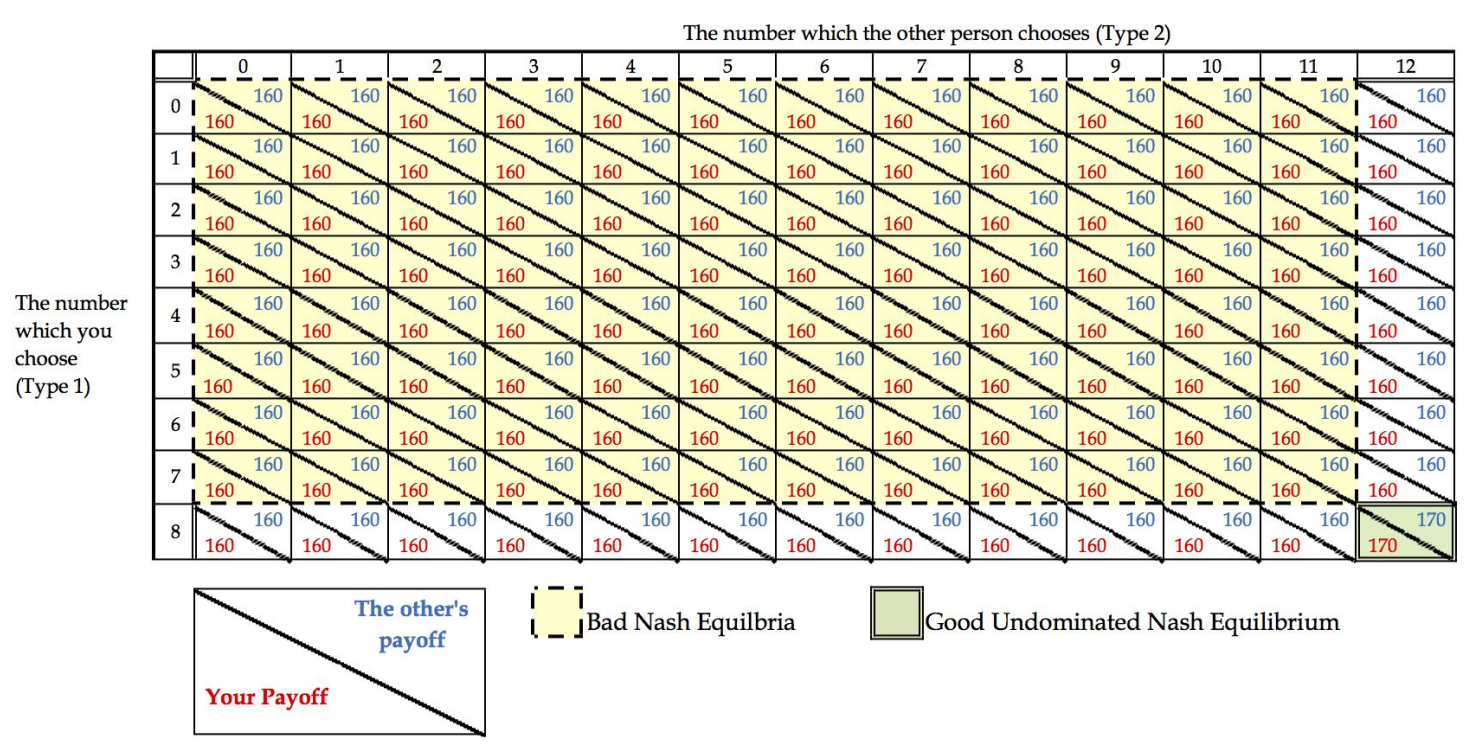

Table 3: Payoff table obtained by eliminating the weakly dominated strategies of two players from Table 1.

original $21 \times 21$ payoff table, we have Table 3 that is the $8 \times 12$ payoff table consisting of eight strategies of Type $1, s_{1} \in\{0, \ldots, 7\}$ and twelve strategies of Type $2, s_{2} \in$ $\{0, \ldots, 11\}$. In Table 3 , there is one "good" Nash equilibria, $\left(s_{1}, s_{2}\right)=(8,12)$, whereas there are ninety-six "bad" Nash equilibria. ${ }^{8}$ Notice that each Type 1's strategy $s_{1} \in$ $\{0, \ldots, 7\}$ is weakly dominated by $s_{1}=8$, and each Type 2's strategy $s_{2} \in\{0, \ldots, 11\}$ is weakly dominated by $s_{2}=12$ in Table 3 . Thus, $(8,12)$ is a unique undominated Nash equilibrium in Table 3 and a unique twice iteratively undominated Nash equilibrium in Table $1 .^{9}$

Moreover, this equilibrium payoff vector $(170,170)$ at $(8,12)$ is Pareto efficient and individually rational and, hence, it is a core payoff vector (Proposition 1). The other good Nash equilibrium payoff vectors in Table $1,(180,160)$ and $(160,180)$, are also

\footnotetext{
${ }^{8}$ The set of "bad" Nash equilibria after eliminating these weakly dominated strategies of two players from the original payoff table coincides with that of "bad" Nash equilibria of the original payoff table mentioned in Footnote 8.

${ }^{9}$ Additionally, $(8,12)$ is a unique pair of strategies surviving after a twice iterated elimination of weakly dominated strategies in Table 1 . In other words, the unique undominated Nash equilibrium coincides with the unique pair of strategies surviving after twice iterated elimination of weakly dominated strategies under our experimental setting. In general, however, the set of twice iteratively undominated Nash equilibria may differ from the set of pairs of strategies surviving after twice iterated elimination of weakly dominated strategies. We need the concept of twice iteratively undominated Nash equilibria to obtain the core implementation result in Proposition 1. See Appendix B for more details.
} 
Pareto efficient and individually rational, and they are core payoff vectors. All bad Nash equilibrium payoff vectors in Table 1 are the same, $(160,160)$, and they are individually rational but not Pareto efficient.

\subsection{Treatment $\mathrm{P}$}

Let the strategy space of each type be the set of integers from 0 to 20 . According to the rules of the pivotal mechanism described in Section 3.5, we can construct the payoff tables of Types 1 and 2. The payoff tables that we use in Treatment $\mathrm{P}$ are Tables 4 and 5 whose structures are the same as the original payoff tables except that a linear transformation of the utility functions is employed: $10 u_{i}-40$ for each type $i \in\{1,2\}$.

Table 4 (resp. Table 5) is a payoff table of Type 1 (resp. Type 2) with both types' payoffs displayed. Table 4 also specifies the dominant strategy equilibria and the other Nash equilibria. ${ }^{10}$ Type 1's dominant strategies are 8 and 9, and Type 2's dominant strategies are 12 and 13. The two dominant strategies are equivalent for each type in that their own payoffs are identical for every possible strategy played by the other type. In this sense, there is an essentially unique dominant strategy for each type. However, the payoffs of Type 2 are different depending on Type 1's choices: Type 2's payoff is 170 when Type 1 selects 8 and Type 2 chooses 12 or 13, whereas Type 2's payoff is 180 when Type 1 selects 9 and Type 2 chooses 12 or 13 .

There is a huge set of Nash equilibria in Table $4 .{ }^{11}$ The lower-right region of Nash equilibria is "good" in the sense that the public project is undertaken and, hence, decision efficiency is satisfied. The upper-left region of Nash equilibria is "bad" in the sense that the public project is not undertaken and, thus, decision efficiency is not satisfied. The number of good Nash equilibria is 137, whereas the number of bad Nash equilibria is 96. Implementation is clearly not secure in the sense of Cason et al. (2006).

Notice that the payoff vector $\left(u_{1}, u_{2}\right)$ is Pareto efficient if $u_{1}+u_{2}=340$, and it is individually rational if $u_{1} \geq 160$ and $u_{2} \geq 160$ in the current environment. The dominant strategy equilibrium payoffs, $(150,170)$ and $(150,180)$, are neither Pareto

\footnotetext{
${ }^{10}$ Again, in the payoff table that we actually distributed to subjects, there was no tag and highlighting indicating the equilibria, as in Table 4 .

${ }^{11}$ This is because the best response function of each type has a "flat" structure. For instance, (i) when Type 2 selects 7 , the payoffs of Type 1 are "high" (160) if Type 1 chooses less than or equal to 12 , and those are "low" (120) otherwise; (ii) when Type 2 chooses 11, the payoffs of Type 1 are the same (150) for all Type 1's strategies; and (iii) when Type 2 selects 16, the payoffs of Type 1 are "low" (100) if Type 1 chooses less than or equal to 3, and Type 1's payoffs are "high" (150) otherwise. In other words, given each strategy of the other type, either a) the payoffs of each type are divided into just two "tiers": a "high" payoff obtained by choosing "good" strategies and a "low" payoff by "bad" strategies, or b) the payoffs are the same for all strategies.
} 


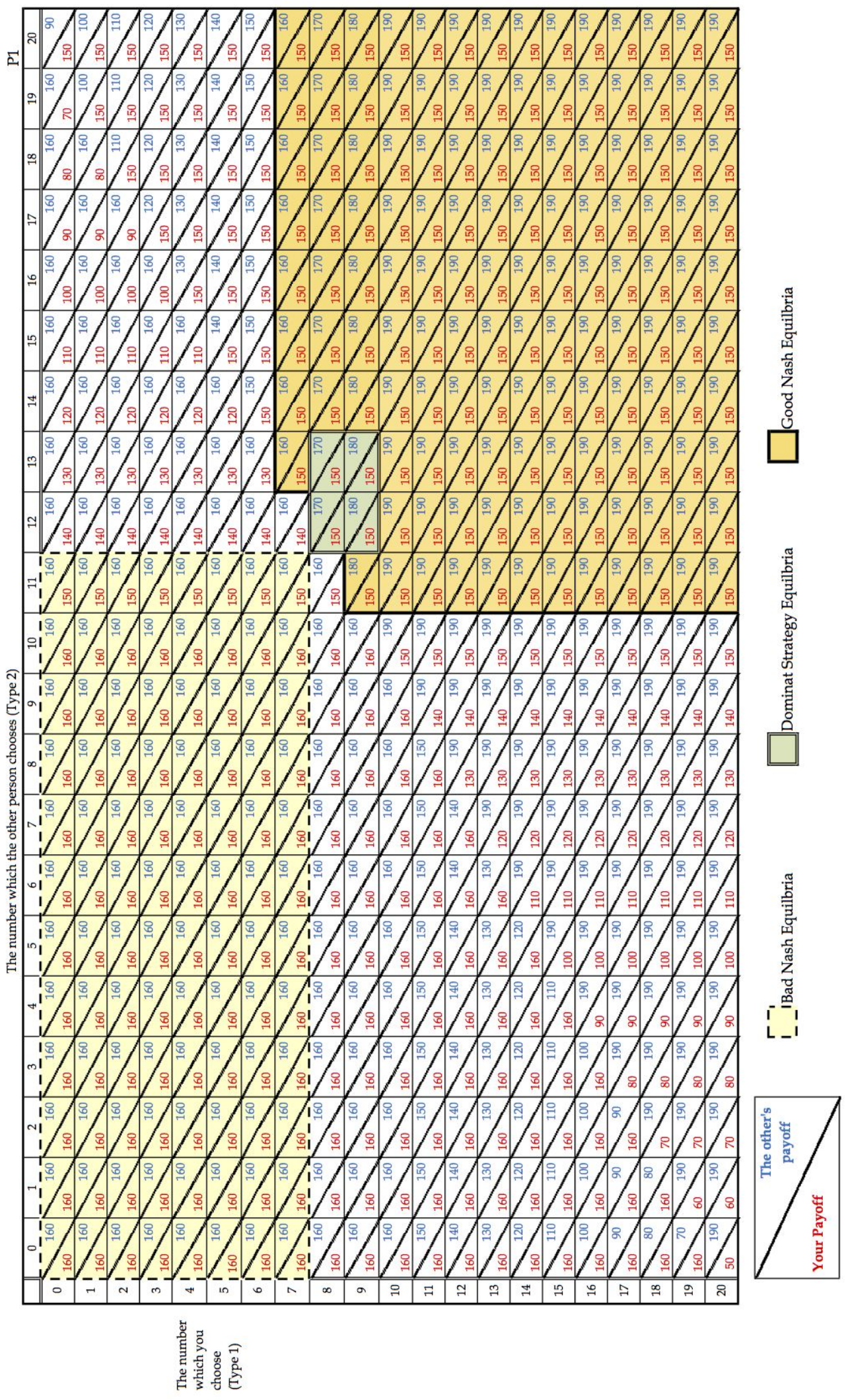

Table 4: Payoff table of Type 1 in Treatment P. 


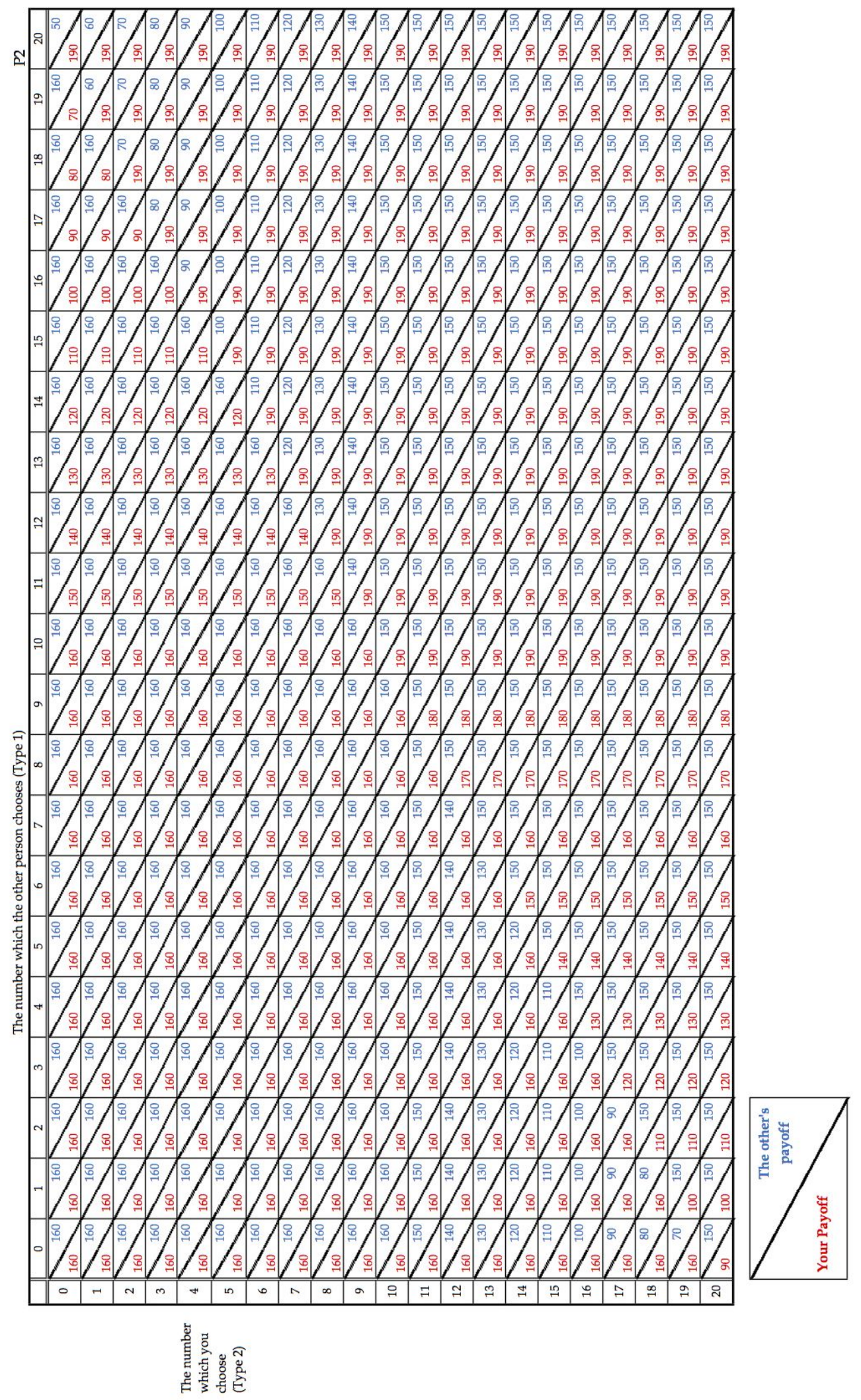

Table 5: Payoff table of Type 2 in Treatment P. 
efficient nor individually rational. This is because the pivotal mechanism generates a wasteful budget surplus, that is, the tax revenue exceeds the cost of undertaking the public project. It is easy to check that the ratio of Pareto efficient payoffs among the good Nash equilibrium payoffs is $80.3 \%\left(=\frac{110}{137}\right)$, but no good Nash equilibrium payoff is individually rational. The ratio of individually rational payoffs among the

bad Nash equilibrium payoffs is $91.7 \%\left(=\frac{88}{96}\right)$, but no bad Nash equilibrium payoff is Pareto efficient.

Although no Nash equilibrium payoff vector belongs to the core, the pivotal mechanism is designed specifically to achieve decision efficiency rather than Pareto efficiency or individual rationality. Since decision efficiency has played a central role in the literature, we check whether the outcome is consistent with decision efficiency in our experiment.

\subsection{Procedures}

We conducted two sessions in each of the two treatments at Tokyo Institute of Technology during January and June of 2013 and July of 2014. Twenty subjects participated in each session (eighty separate subjects in total). We recruited the student subjects by campus-wide advertisement. These students were told that there would be an opportunity to earn money in a research experiment. None of them had prior experience in a public project experiment. No subject attended more than one session. Each session took approximately two hours to complete. The mean payoff per subject was $\$ 32.57$ $(\$ 1=100$ yen $)$ in Treatment $\mathrm{P}$ and $\$ 33.56$ in Treatment V.

In each session, twenty subjects were seated at computer stations that were separated with visual partitions in the Experimental Economics Laboratory at Tokyo Institute of Technology. We made ten pairs out of twenty subjects and conducted twenty periods. In every period, each of Type 1 subjects was paired with one of Type 2 subjects. The pairings were anonymous and were determined in advance by experimenters to pair the same two subjects just two times. Each subject was informed that the person the subject was paired with was randomly chosen by the experimenters and that the person the subject was paired with changed over twenty periods. Each subject could not know which person the subject was paired with at each period.

Each subject received written instructions, a record sheet, and a payoff table. Each subject chose an integer number between 0 and 20 by looking at their own payoff table only. In each subject's payoff table, both their own payoffs and the payoffs of the other type were shown in Treatments $\mathrm{P}$ and $\mathrm{V}$. 
After deciding which number to choose, each subject typed in that number into the computer. We used the z-Tree program (Urs Fischbacher, 2007). After the calculation of payoffs, the following information was displayed on each subject's computer screen:

- the subject's chosen number;

- the other's chosen number;

- the subject's own payoff; and

- the other's payoff.

Each subject was asked to fill out these values as well as the reasons why they had chosen a particular number. These steps were repeated for twenty periods. No information or decisions regarding the other pairs were shown on the computer screen. No communication among the subjects was allowed, and we declared that the experiment would be stopped if we observed any communication among the subjects. This did not happen.

Before the real twenty periods, the subjects had an opportunity to practice in two periods using a payoff table that differed from the table employed in the actual experiment. In these non-monetary periods, the numbers to be chosen were decided in advance by the experimenters. We allowed the subjects ten minutes to examine the payoff table before the real periods started.

\section{Results}

Since each period had 20 pairs of subjects and each session had 20 periods, there were 400 pairs of data. We denote each pair by $\left(s_{1}, s_{2}\right)$, where $s_{i}$ is a strategy chosen by a subject of Type $i \in\{1,2\}$.

\subsection{Treatment V}

Figure 1 shows the frequency of distribution of all data in Treatment $\mathrm{V}$. The maximum frequency pair was the unique twice iteratively undominated Nash equilibrium $(8,12)$ with 313 pairs out of 400 , the second frequency pair was $(8,10)$ with 31 pairs, the third frequency pair was $(8,11)$ with 11 pairs, and the fourth frequency pair was $(7,12)$ with 6 pairs. The total frequency of Nash equilibria other than $(8,12)$ was 8 . All of them were bad Nash equilibria that failed to achieve decision efficiency. The total 


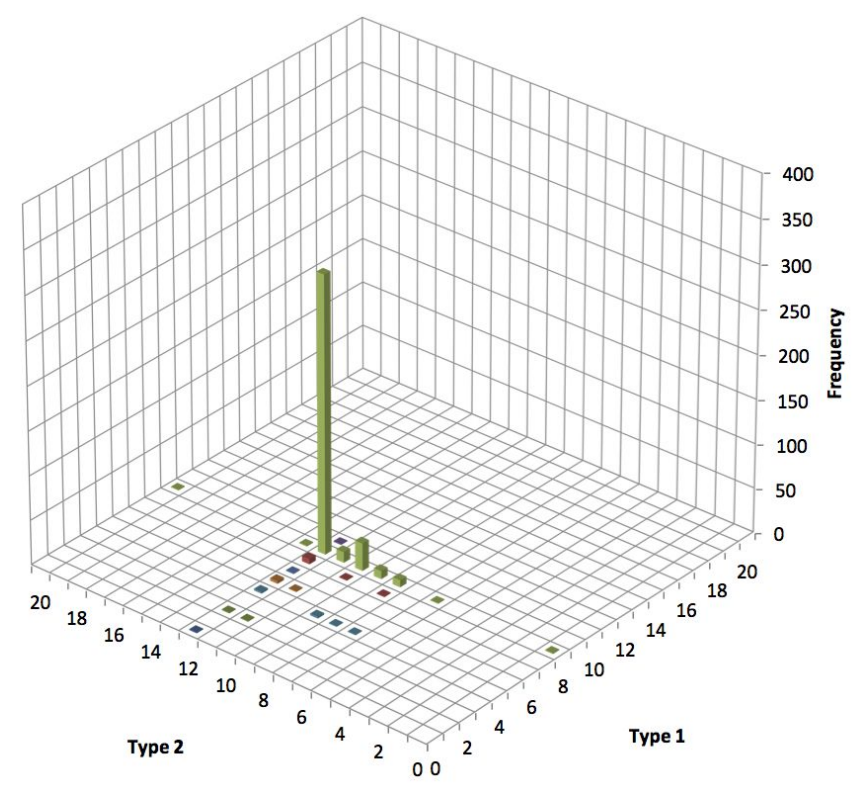

Figure 1: All pairs choices in Treatment V.

frequency of strategy profiles that achieved decision efficiency was 316 . The maximum frequency strategy chosen by Type 1 subjects was $s_{1}=8$ with 377 choices out of 400 , the second maximum frequency strategy was $s_{1}=7$ with 8 choices, and the third maximum frequency strategy was $s_{1}=4$ with 6 choices. The maximum frequency strategy chosen by Type 2 subjects was $s_{2}=12$ with 328 choices out of 400 , the second was $s_{2}=10$ with 32 choices, and the third was $s_{1}=11$ with 14 choices.

Figure 2 displays the rates that Type 1 and Type 2 subjects chose $s_{1}=8$ and $s_{2}=12$, respectively. After period 3, the ratios of Type 1 subjects who chose $s_{1}=8$ were at least $90 \%$. The ratios of Type 2 subjects who chose $s_{2}=12$ were at least $80 \%$ after period 4. Figure 2 also demonstrates the rates that pairs of subjects played the unique twice iteratively undominated Nash equilibrium $(8,12)$ for all periods. These equilibrium rates were at least $70 \%$ after period 4 . By conducting the Wilcoxon signed rank tests period by period, we found that Type 1 subjects' median choice was not significantly different from 8 in 18 out of 20 periods (periods $3-20$ at the $5 \%$ significance level) and that Type 2 subjects' median choice was not significantly different from 12 in 18 periods (periods 2 and $4-20$ at the $5 \%$ significance level).

These results are summarized by the following observations.

\section{Observation 1.}

(i) The frequency of the unique twice iteratively undominated Nash equilibrium was 


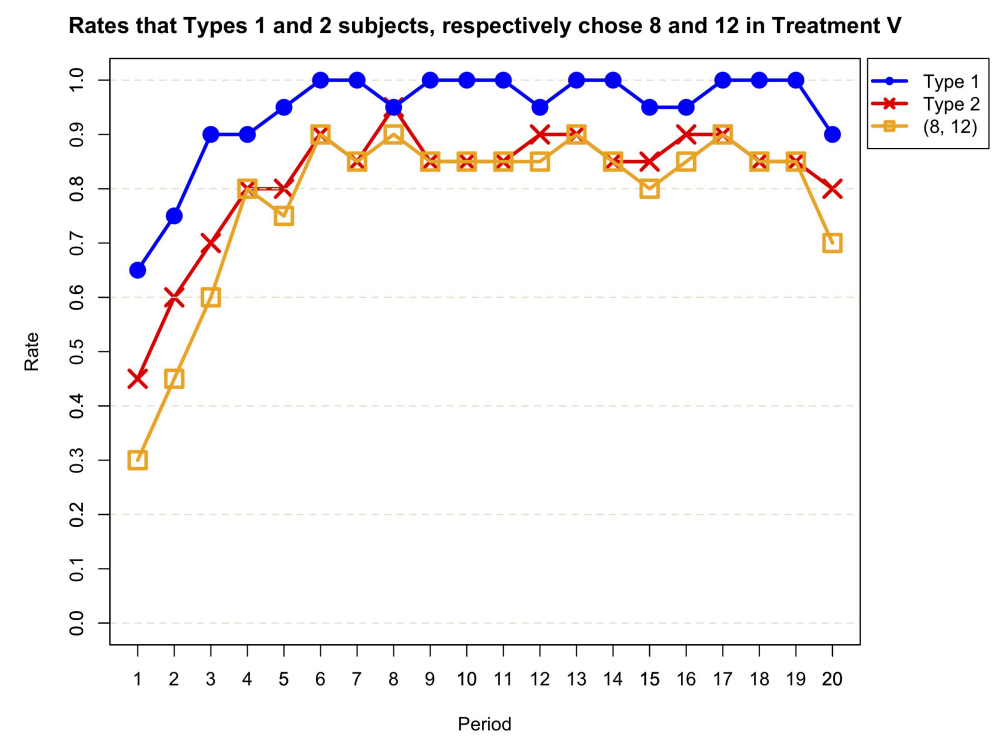

Figure 2: Rates that Types 1 and 2 subjects, respectively, chose $s_{1}=8$ and $s_{2}=12$ in Treatment V.

$78 \%\left(=\frac{313}{400}\right)$ across all periods in Treatment $\mathrm{V}$.

(ii) The frequency that Type 1 subjects chose $s_{1}=8$ was $94 \%\left(=\frac{377}{400}\right)$ across all periods in Treatment $\mathrm{V}$.

(iii) The frequency that Type 2 subjects chose $s_{2}=12$ was $82 \%\left(=\frac{328}{400}\right)$ across all periods in Treatment $\mathrm{V}$.

\subsection{Treatment $\mathbf{P}$}

Figure 3 shows the frequency of distribution of all data in Treatment $\mathrm{P}$. The maximum frequency pair was $(8,13)$ with 95 pairs out of 400 , the second was $(8,12)$ with 91 pairs, the third was $(9,12)$ with 30 pairs, the fourth was $(10,12)$ with 29 pairs, and the fifth was $(9,13)$ with 26 pairs. The total frequency of dominant strategy equilibria was 242 . The total frequency of Nash equilibria was 370. All Nash equilibria were good. The maximum frequency strategy chosen by Type 1 subjects was $s_{1}=8$ with 224 choices out of 400 , the second was $s_{1}=9$ with 72 choices, and the third was $s_{1}=10$ with 70 choices. The total frequency of dominant strategies selected by Type 1 subjects was 296. The maximum frequency strategy chosen by Type 2 was $s_{2}=12$ with 167 choices out of 400 , the second was $s_{2}=13$ with 160 choices, and the third was $s_{1}=20$ with 15 


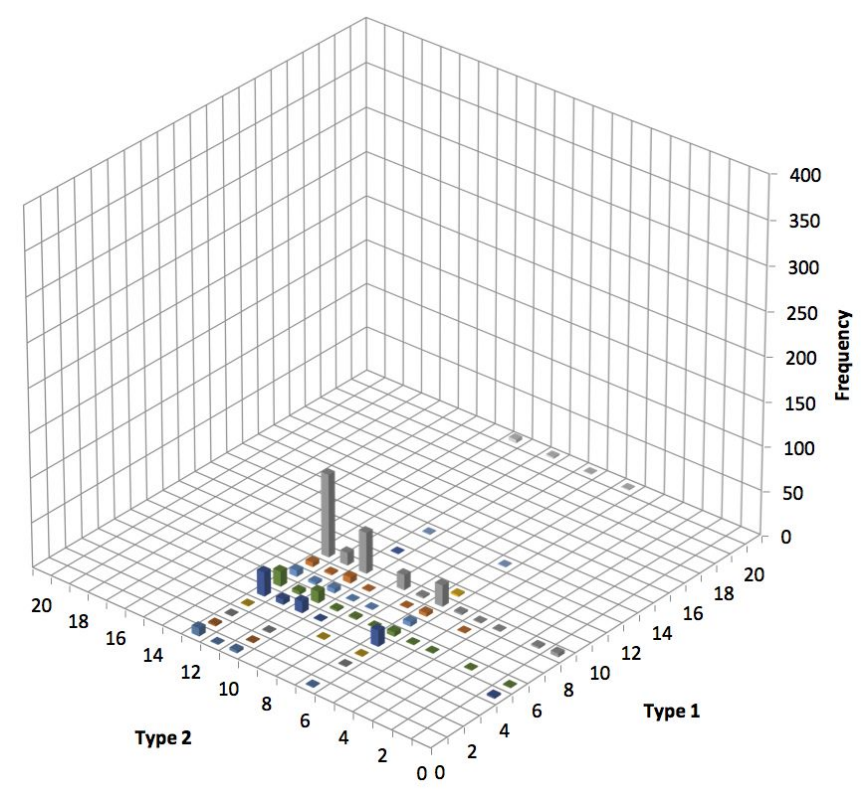

Figure 3: All pairs choices in Treatment P.

choices. Hence, the total frequency of dominant strategies selected by Type 2 subjects was 327 .

Figure 4 displays the rates that each type of subjects chose dominant strategies separately as well as the rates that pairs of subjects played dominant strategy equilibria for all periods.

These results are summarized by the following observations.

\section{Observation 2.}

(i) The frequency of dominant strategy equilibria was $61 \%\left(=\frac{242}{400}\right)$ across all periods in Treatment $\mathrm{P}$.

(ii) The frequency of good Nash equilibria was $93 \%\left(=\frac{370}{400}\right)$ across all periods in Treatment P.

(iii) The frequency that Type 1 subjects chose dominant strategies was $74 \%\left(=\frac{296}{400}\right)$ across all periods in Treatment $\mathrm{P}$.

(iv) The frequency that Type 2 subjects chose dominant strategies was $82 \%\left(=\frac{327}{400}\right)$ across all periods in Treatment $\mathrm{P}$. 


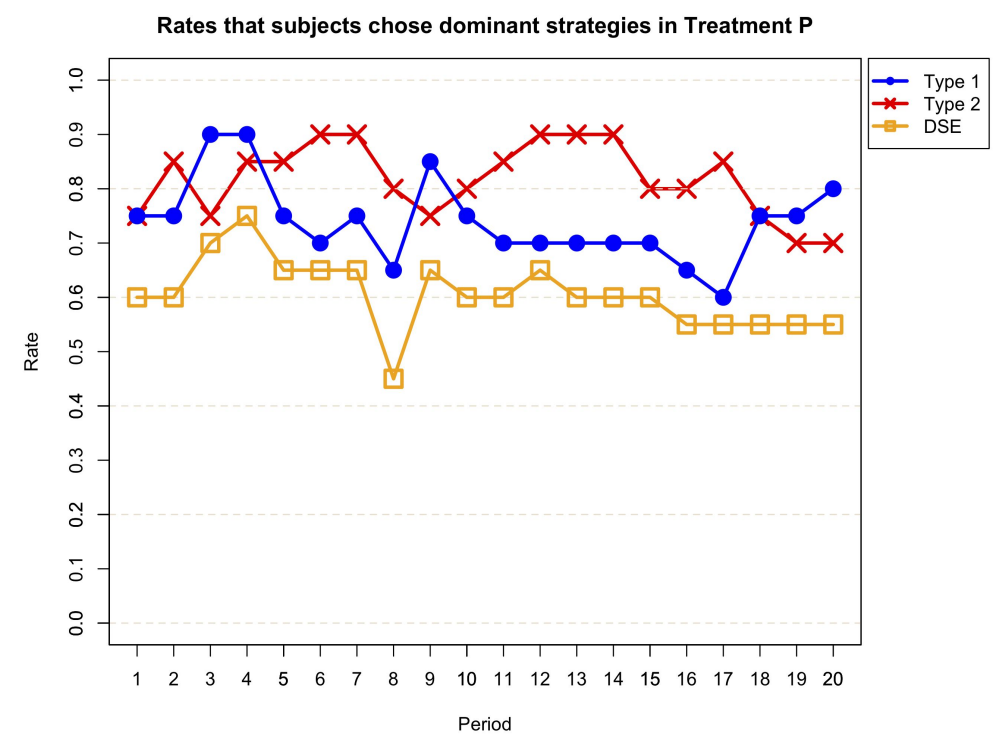

Figure 4: Rates that subjects chose dominant strategies in Treatment P.

\subsection{Comparing the two mechanisms}

We compare the performances of the voluntary contribution mechanism and those of the pivotal mechanism based on the following four indices:

- Pareto efficiency index: We define the Pareto efficiency index at each period as the ratio of Pareto efficient outcomes at that period. The sum of two subjects' payoffs in one pair is equal to 340 at any Pareto efficient outcome.

- Individual rationality index: We define the individual rationality index at each period as the ratio of individually rational outcomes at that period such that each subject receives a payoff at least as large as their own payoff before participating in the mechanism (160), that is, the payoff the subject receives from their initial endowment of the private good with no public project.

- Surplus index: We define the surplus index by

$$
\text { Surplus Index }=\frac{\text { average of sums of two subjects' payoffs }- \text { reference payoff }}{\text { Pareto efficient payoff }- \text { reference payoff }},
$$

where the Pareto efficient payoff is the sum of two subjects' payoffs at any Pareto efficient outcome, which equals 340, and the reference payoff is the sum of two subjects' payoffs that they receive before participating in the mechanism, which 
equals 320. This index measures how much gain subjects obtain by participating in a mechanism.

- Decision efficiency index: We define the decision efficiency index at each period as the ratio of outcomes at which the public project is undertaken at that period.

Figure 5 displays the mean Pareto efficiency index, the mean individual rationality index, the mean surplus index, and the mean decision efficiency index at each period in Treatments $\mathrm{V}$ and $\mathrm{P}$. As shown in this figure, we observed that the mean Pareto efficiency index, the mean individual rationality index, and the surplus index for Treatment $\mathrm{V}$ were higher than those for Treatment $\mathrm{P}$ in each period (except for the first period for the surplus index). We also observed that the mean decision efficiency index for Treatment $\mathrm{P}$ was higher than that for Treatment $\mathrm{V}$ in 18 out of 20 periods (except for periods 8 and 11), although the mean decision efficiency index for Treatment $\mathrm{V}$ attained at least $80 \%$ in 16 out of 20 periods (except for periods $1-3$ and 20 ).

To formally test these graphical observations, we ran the panel regressions. Table 6 reports the panel regression results of the pooled data. Among the four regressions, the random effects panel probit model was applied in the regressions of the Pareto efficiency index, the individual rationality index, and the decision efficiency index, while the random effects panel model was conducted for the regression of the surplus index. Besides the continuous variable of Period controlling for round effects, the dummy variable of Treatment $P$ was included in the four regressions as the independent variable, leaving Treatment $\mathrm{V}$ as the base of comparison. As presented in the table, the dummy variable of Treatment $P$ was estimated with significantly negative sign in the regressions of the Pareto efficiency index, the individual rationality index, and the surplus index, implying that the voluntary contribution mechanism is superior to the pivotal mechanism based on these criteria. However, this variable was significantly and positively estimated in the regression of the decision efficiency index, which exhibits a better performance of the pivotal mechanism than the voluntary contribution mechanism in the sense of decision efficiency.

These results are summarized by the following observations.

\section{Observation 3.}

(i) The Pareto efficiency index for Treatment $\mathrm{V}$ was significantly higher than the Pareto efficiency index for Treatment P. 


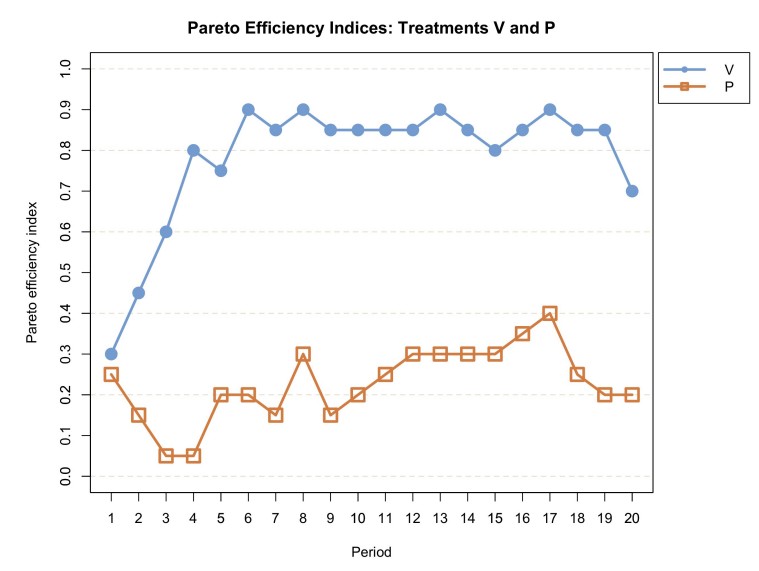

(a) Pareto efficiency index

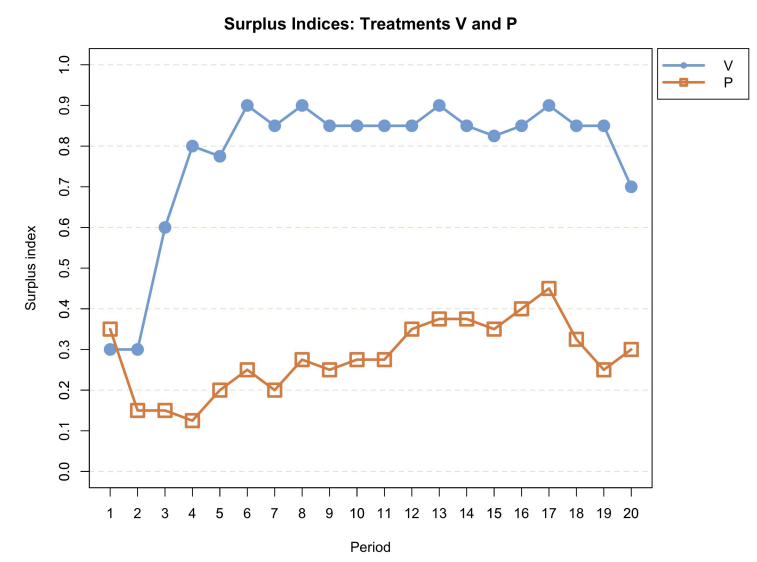

(c) Surplus index

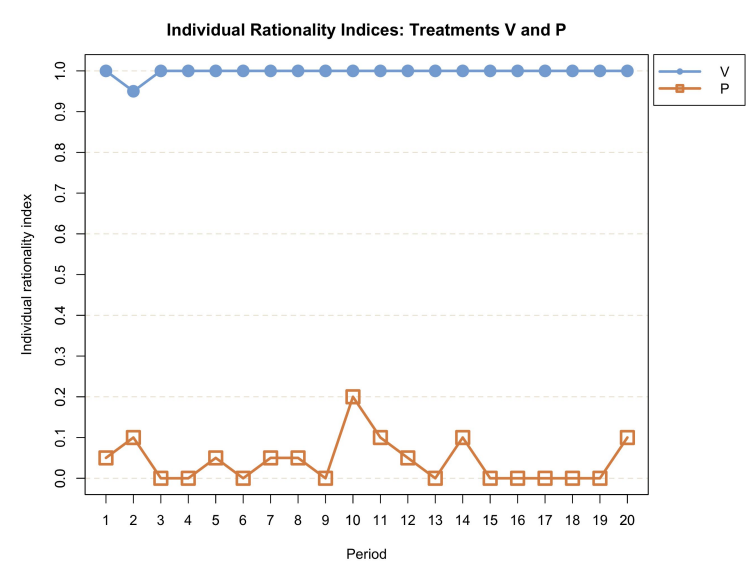

(b) Individual rationality index

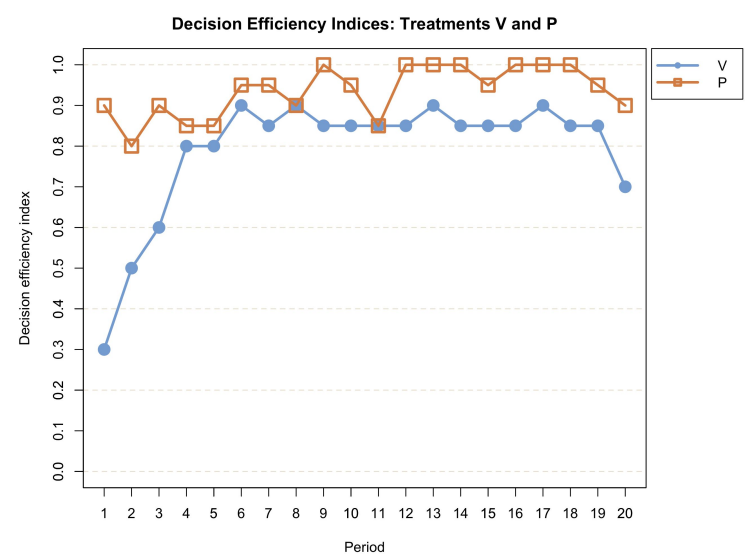

(d) Decision efficiency index

Figure 5: Comparing the voluntary contribution mechanism and the pivotal mechanism based on four indices.

(ii) The individual rationality index for Treatment $\mathrm{V}$ was significantly higher than the individual rationality index for Treatment $\mathrm{P}$.

(iii) The surplus index for Treatment $\mathrm{V}$ was significantly higher than the surplus index for Treatment P.

(iv) The decision efficiency index for Treatment $\mathrm{V}$ was significantly lower than the decision efficiency index for Treatment $P$. 


\begin{tabular}{ccccc}
\hline & \multicolumn{4}{c}{ Index } \\
\cline { 2 - 5 } & $\begin{array}{c}\text { Pareto } \\
\text { efficiency }\end{array}$ & $\begin{array}{c}\text { Individual } \\
\text { rationality }\end{array}$ & Surplus & $\begin{array}{c}\text { Decision } \\
\text { efficiency }\end{array}$ \\
\hline Treatment $P$ & $-2.012^{* *}$ & $-4.662^{* *}$ & $-0.494^{* *}$ & $0.747^{* *}$ \\
& $(0.448)$ & $(0.300)$ & $(0.069)$ & $(0.262)$ \\
Period & $0.046^{*}$ & -0.012 & $0.013^{*}$ & $0.049^{*}$ \\
& $(0.020)$ & $(0.022)$ & $(0.006)$ & $(0.023)$ \\
Constant & 0.411 & $2.969^{* *}$ & $0.640^{* *}$ & 0.333 \\
& $(0.461)$ & $(0.408)$ & $(0.112)$ & $(0.461)$ \\
\hline Log-likelihood & -365.671 & -70.720 & & -289.310 \\
$R^{2}$ (overall) & \multicolumn{4}{c}{0.248} \\
Observations & 800 & 800 & 800 & 800 \\
Notes: & ${ }^{* *} p<0.001,{ }^{*} p<0.01$. Standard errors in the parentheses are clustered in session.
\end{tabular}

Table 6: Panel regressions on the four indices

\section{Concluding remarks}

In our experiment, we used the payoff tables to simplify the presentation of the two mechanisms for subjects. We provided no explanation regarding the rules of the mechanisms or how the payoff tables were constructed. The rule of the pivotal mechanism is more complicated than that of the voluntary contribution mechanism. Using payoff tables only allows for a comparison of the two mechanisms holding their degree of transparency constant. We selected maximally-transparent conditions as a first step in this initial experiment, but payoff tables are somewhat unrealistic for potential applications of these mechanisms in the field. Hence, we need to conduct another experiment to check whether the voluntary contribution mechanism performs better than the pivotal mechanism when the rules of the two mechanisms are explained. Since the rule of the pivotal mechanism is more difficult to understand, it is natural to expect that the voluntary contribution mechanism would perform better than the pivotal mechanism when the rules of the mechanisms are explained. This is left for future experiments.

Several other questions remain to be answered. We do not consider incomplete information in which all agents know their own true values, but not the values to others. In contrast to the complete information setting, the theoretical predictions suggest that the pivotal mechanism might perform better than the voluntary contribution mechanism under incomplete information. Thus, it would be worth investigating the performances of these mechanisms under incomplete information by economic experiments. Moreover, there were three or more agents in each group in some existing experiments 
(e.g., Bagnoli and McKee (1991), Marks and Croson (1999), and Attiyeh et al. (2000)), whereas there were two subjects in each group in our experiment. Therefore, it is worthwhile to compare experimentally the performance of the voluntary contribution mechanism and that of the pivotal mechanism when there are more than two subjects in each group. We hope to address these issues in future work. 


\section{A Appendix: Proofs}

\section{A.1 Proof of Fact 1}

The "if" part. Suppose, by contradiction, that $(y, t) \notin P(v)$. Then, there is $\left(y^{\prime}, t^{\prime}\right) \in$ $A$ such that $u_{i}\left(y^{\prime}, t_{i}^{\prime} ; v_{i}\right) \geq u_{i}\left(y, t_{i} ; v_{i}\right)$ for each $i \in\{1,2\}$ with a strict inequality for some $i \in\{1,2\}$. There are two cases.

- Case 1: $v_{1}+v_{2} \geq c$. Then,

$$
u_{1}\left(y^{\prime}, t_{1}^{\prime} ; v_{1}\right)+u_{2}\left(y^{\prime}, t_{2}^{\prime} ; v_{2}\right)>u_{1}\left(y, t_{1} ; v_{1}\right)+u_{2}\left(y, t_{2} ; v_{2}\right)=v_{1}+v_{2}-c+\omega_{1}+\omega_{2} \text {. }
$$

If $y^{\prime}=1$, then

$$
\begin{aligned}
v_{1}+v_{2}+\omega_{1}+\omega_{2}+t_{1}^{\prime}+t_{2}^{\prime} & =u_{1}\left(y^{\prime}, t_{1}^{\prime} ; v_{1}\right)+u_{2}\left(y^{\prime}, t_{2}^{\prime} ; v_{2}\right) \\
& >u_{1}\left(y, t_{1} ; v_{1}\right)+u_{2}\left(y, t_{2} ; v_{2}\right) \\
& =v_{1}+v_{2}-c+\omega_{1}+\omega_{2}
\end{aligned}
$$

which implies $-\left(t_{1}^{\prime}+t_{2}^{\prime}\right)<c$, a contradiction. If $y^{\prime}=0$, then

$$
\begin{aligned}
\omega_{1}+\omega_{2}+t_{1}^{\prime}+t_{2}^{\prime} & =u_{1}\left(y^{\prime}, t_{1}^{\prime} ; v_{1}\right)+u_{2}\left(y^{\prime}, t_{2}^{\prime} ; v_{2}\right) \\
& >u_{1}\left(y, t_{1} ; v_{1}\right)+u_{2}\left(y, t_{2} ; v_{2}\right) \\
& =v_{1}+v_{2}-c+\omega_{1}+\omega_{2},
\end{aligned}
$$

which implies $-\left(t_{1}^{\prime}+t_{2}^{\prime}\right)<c-\left(v_{1}+v_{2}\right) \leq 0$, a contradiction.

- Case 2: $v_{1}+v_{2}<c$. Then,

$$
u_{1}\left(y^{\prime}, t_{1}^{\prime} ; v_{1}\right)+u_{2}\left(y^{\prime}, t_{2}^{\prime} ; v_{2}\right)>u_{1}\left(y, t_{1} ; v_{1}\right)+u_{2}\left(y, t_{2} ; v_{2}\right)=\omega_{1}+\omega_{2}
$$

If $y^{\prime}=1$, then

$$
\begin{aligned}
v_{1}+v_{2}+\omega_{1}+\omega_{2}+t_{1}^{\prime}+t_{2}^{\prime} & =u_{1}\left(y^{\prime}, t_{1}^{\prime} ; v_{1}\right)+u_{2}\left(y^{\prime}, t_{2}^{\prime} ; v_{2}\right) \\
& >u_{1}\left(y, t_{1} ; v_{1}\right)+u_{2}\left(y, t_{2} ; v_{2}\right) \\
& =\omega_{1}+\omega_{2}
\end{aligned}
$$

which implies $-\left(t_{1}^{\prime}+t_{2}^{\prime}\right)<v_{1}+v_{2}<c$, a contradiction. If $y^{\prime}=0$, then

$$
\omega_{1}+\omega_{2}+t_{1}^{\prime}+t_{2}^{\prime}=u_{1}\left(y^{\prime}, t_{1}^{\prime} ; v_{1}\right)+u_{2}\left(y^{\prime}, t_{2}^{\prime} ; v_{2}\right)
$$




$$
\begin{aligned}
& >u_{1}\left(y, t_{1} ; v_{1}\right)+u_{2}\left(y, t_{2} ; v_{2}\right) \\
& =\omega_{1}+\omega_{2}
\end{aligned}
$$

which implies $-\left(t_{1}^{\prime}+t_{2}^{\prime}\right)<0$, a contradiction.

The "only if" part. Let $(y, t) \in P(v)$. There are three cases.

- Case 1: $\boldsymbol{v}_{\mathbf{1}}+\boldsymbol{v}_{\mathbf{2}}>\boldsymbol{c}$. In this case, it suffices to show that $y=1$, because this together with $(y, t) \in P(v)$ implies that $u_{1}\left(y, t_{1} ; v_{1}\right)+u_{2}\left(y, t_{2} ; v_{2}\right)=v_{1}+v_{2}-c+\omega_{1}+\omega_{2}$. To show this, suppose by contradiction that $y=0$. Then, $u_{1}\left(y, t_{1} ; v_{1}\right)=\omega_{1}+t_{1}$, $u_{2}\left(y, t_{2} ; v_{2}\right)=\omega_{2}+t_{2}$, and $-\left(t_{1}+t_{2}\right) \geq 0$. Without loss of generality, assume $t_{1} \geq t_{2}$. Let $\left(y^{\prime}, t^{\prime}\right) \in A$ be such that $y^{\prime}=1,-\left(t_{1}^{\prime}+t_{2}^{\prime}\right)=c$, and $t_{1}^{\prime}=t_{1}-v_{1}$. Since $v_{1}+v_{2}>c$, $v_{1}+v_{2}-c=v_{1}+v_{2}+\left(t_{1}^{\prime}+t_{2}^{\prime}\right)>0 \geq t_{1}+t_{2}$, which implies that $v_{2}+t_{2}^{\prime}>t_{2}$. It then follows that

$$
\begin{aligned}
& u_{1}\left(y^{\prime}, t_{1}^{\prime} ; v_{1}\right)=v_{1}+t_{1}^{\prime}+\omega_{1}=t_{1}+\omega_{1}=u_{1}\left(y, t_{1} ; v_{1}\right), \\
& u_{2}\left(y^{\prime}, t_{2}^{\prime} ; v_{2}\right)=v_{2}+t_{2}^{\prime}+\omega_{1}>t_{2}+\omega_{2}=u_{2}\left(y, t_{2} ; v_{2}\right),
\end{aligned}
$$

which contradict $(y, t) \in P(v)$.

- Case 2: $\boldsymbol{v}_{\mathbf{1}}+\boldsymbol{v}_{\mathbf{2}}<\boldsymbol{c}$. In this case, it suffices to show that $y=0$, because this together with $(y, t) \in P(v)$ implies that $u_{1}\left(y, t_{1} ; v_{1}\right)+u_{2}\left(y, t_{2} ; v_{2}\right)=\omega_{1}+\omega_{2}$. To show this, suppose by contradiction that $y=1$. Then, $u_{1}\left(y, t_{1} ; v_{1}\right)=v_{1}+\omega_{1}+t_{1}$, $u_{2}\left(y, t_{2} ; v_{2}\right)=v_{2}+\omega_{2}+t_{2}$, and $-\left(t_{1}+t_{2}\right) \geq c>0$. Without loss of generality, assume $v_{1}+t_{1} \geq v_{2}+t_{2}$. Let $\left(y^{\prime}, t^{\prime}\right) \in A$ be such that $y^{\prime}=0, t_{1}^{\prime}+t_{2}^{\prime}=0$, and $t_{1}^{\prime}=v_{1}+t_{1}$. Since $v_{1}+v_{2}<c, t_{1}^{\prime}+t_{2}^{\prime}=0>v_{1}+v_{2}-c \geq v_{1}+v_{2}+\left(t_{1}+t_{2}\right)$, which implies that $t_{2}^{\prime}>v_{2}+t_{2}$. It then follows that

$$
\begin{aligned}
& u_{1}\left(y^{\prime}, t_{1}^{\prime} ; v_{1}\right)=\omega_{1}+t_{1}^{\prime}=v_{1}+t_{1}+\omega_{1}=u_{1}\left(y, t_{1} ; v_{1}\right), \\
& u_{2}\left(y^{\prime}, t_{2}^{\prime} ; v_{2}\right)=\omega_{1}+t_{2}^{\prime}>v_{2}+t_{2}+\omega_{2}=u_{2}\left(y, t_{2} ; v_{2}\right),
\end{aligned}
$$

which contradict $(y, t) \in P(v)$.

- Case 3: $\boldsymbol{v}_{\mathbf{1}}+\boldsymbol{v}_{\mathbf{2}}=\boldsymbol{c}$. Then, either $y=1$ or $y=0$. If $y=1$, then it follows from $(y, t) \in P(v)$ that $u_{1}\left(y, t_{1} ; v_{1}\right)+u_{2}\left(y, t_{2} ; v_{2}\right)=v_{1}+v_{2}-c+\omega_{1}+\omega_{2}$. If $y=0$, then it follows from $(y, t) \in P(v)$ that $v_{1}+v_{2}=c$, that $u_{1}\left(y, t_{1} ; v_{1}\right)+u_{2}\left(y, t_{2} ; v_{2}\right)=\omega_{1}+\omega_{2}=$ $v_{1}+v_{2}-c+\omega_{1}+\omega_{2}$. 


\section{A.2 Proof of Fact 2}

We prove this fact by contraposition. Suppose that an allocation $(y, t) \in A$ is not decision efficient for $v$. Then, $y \notin \arg \max _{y^{\prime} \in\{0,1\}}\left(v_{1}+v_{2}\right) \cdot y^{\prime}-c \cdot y^{\prime}$. There are two cases.

- Case 1: $y=1$. Then,

$$
0>v_{1}+v_{2}-c
$$

Let $\left(y^{\prime}, t^{\prime}\right) \in A$ be such that $y^{\prime}=0$ and for each $i \in\{1,2\}$,

$$
t_{i}^{\prime} \equiv t_{i}+v_{i}+\frac{c-\left(v_{1}+v_{2}\right)}{2}
$$

Note that by $-\left(t_{1}+t_{2}\right) \geq c$,

$$
\begin{aligned}
t_{1}^{\prime}+t_{2}^{\prime} & =t_{1}+t_{2}+v_{1}+v_{2}+c-\left(v_{1}+v_{2}\right) \\
& =t_{1}+t_{2}+c \\
& \leq 0
\end{aligned}
$$

By (2), we have

$$
\begin{aligned}
& u_{1}\left(y^{\prime}, t_{1}^{\prime} ; v_{1}\right)=\omega_{1}+t_{1}^{\prime}=\omega_{1}+t_{1}+v_{1}+\frac{c-\left(v_{1}+v_{2}\right)}{2}>\omega_{1}+t_{1}+v_{1}=u_{1}\left(y, t_{1} ; v_{1}\right), \\
& u_{2}\left(y^{\prime}, t_{2}^{\prime} ; v_{2}\right)=\omega_{2}+t_{2}^{\prime}=\omega_{2}+t_{2}+v_{2}+\frac{c-\left(v_{1}+v_{2}\right)}{2}>\omega_{2}+t_{2}+v_{2}=u_{2}\left(y, t_{2} ; v_{2}\right) .
\end{aligned}
$$

These imply that $(y, t) \notin P(v)$.

- Case 2: $y=0$. Then,

$$
v_{1}+v_{2}-c>0
$$

Let $\left(y^{\prime}, t^{\prime}\right) \in A$ be such that $y^{\prime}=1$ and for each $i \in\{1,2\}$,

$$
t_{i}^{\prime} \equiv t_{i}-v_{i}+\frac{v_{1}+v_{2}-c}{2} .
$$

Note that by $-\left(t_{1}+t_{2}\right) \geq 0$,

$$
\begin{aligned}
t_{1}^{\prime}+t_{2}^{\prime} & =t_{1}+t_{2}-v_{1}-v_{2}+v_{1}+v_{2}-c \\
& =t_{1}+t_{2}-c
\end{aligned}
$$




$$
\leq-c
$$

By (3), we have

$$
\begin{aligned}
& u_{1}\left(y^{\prime}, t_{1}^{\prime} ; v_{1}\right)=v_{1}+\omega_{1}+t_{1}^{\prime}=\omega_{1}+t_{1}+\frac{v_{1}+v_{2}-c}{2}>\omega_{1}+t_{1}=u_{1}\left(y, t_{1} ; v_{1}\right), \\
& u_{2}\left(y^{\prime}, t_{2}^{\prime} ; v_{2}\right)=v_{2}+\omega_{2}+t_{2}^{\prime}=\omega_{2}+t_{2}+\frac{v_{1}+v_{2}-c}{2}>\omega_{2}+t_{2}=u_{2}\left(y, t_{2} ; v_{2}\right) .
\end{aligned}
$$

These imply that $(y, t) \notin P(v)$.

\section{A.3 Proof of Fact 3}

The "if" part. Suppose, by contradiction, that $(y, t) \in A$ is not individually rational for $v$. Then, there are $i \in\{1,2\}$ and $\left(y^{\prime}, t_{i}^{\prime}\right) \in A_{i}$ such that $u_{i}\left(y^{\prime}, t_{i}^{\prime} ; v_{i}\right)>u_{i}\left(y, t_{i} ; v_{i}\right)$. There are two cases.

- Case 1: $\boldsymbol{v}_{\boldsymbol{i}} \geq \boldsymbol{c}$. Then, $u_{i}\left(y^{\prime}, t_{i}^{\prime} ; v_{i}\right)>u_{i}\left(y, t_{i} ; v_{i}\right) \geq v_{i}-c+\omega_{i}$. If $y^{\prime}=1$, then $v_{i}+\omega_{i}+t_{i}^{\prime}>v_{i}-c+\omega_{i}$, which implies $-t_{i}^{\prime}<c$, a contradiction. If $y^{\prime}=0$, then $\omega_{i}+t_{i}^{\prime}>v_{i}-c+\omega_{i}$, which implies $-t_{i}^{\prime}<c-v_{i}<0$, a contradiction.

- Case 2: $\boldsymbol{v}_{\boldsymbol{i}}<\boldsymbol{c}$. Then, $u_{i}\left(y^{\prime}, t_{i}^{\prime} ; v_{i}\right)>u_{i}\left(y, t_{i} ; v_{i}\right) \geq \omega_{i}$. If $y^{\prime}=1$, then $v_{i}+\omega_{i}+t_{i}^{\prime}>\omega_{i}$, which implies $-t_{i}^{\prime}<v_{i}<c$, a contradiction. If $y^{\prime}=0$, then $\omega_{i}+t_{i}^{\prime}>\omega_{i}$, which implies $-t_{i}^{\prime}<0$, a contradiction.

The "only if" part. We prove this by contraposition. Suppose that there are $(y, t) \in$ $A$ and $i \in\{1,2\}$ such that (1) does not hold. If $v_{i} \geq c$, then $u_{i}\left(y, t_{i} ; v_{i}\right)<v_{i}-c+\omega_{i}$. Let $\left(y^{\prime}, t_{i}^{\prime}\right) \in A_{i}$ be such that $y^{\prime}=1$ and $t_{i}^{\prime}=-c$. Then, $u_{i}\left(y, t_{i} ; v_{i}\right)<u_{i}\left(y^{\prime}, t_{i}^{\prime} ; v_{i}\right)$, which implies $(y, t) \notin I(v)$. If $v_{i}<c$, then $u_{i}\left(y, t_{i} ; v_{i}\right)<\omega_{i}$. Let $\left(y^{\prime}, t_{i}^{\prime}\right) \in A_{i}$ be such that $y^{\prime}=0$ and $t_{i}^{\prime}=0$. Then, $u_{i}\left(y, t_{i} ; v_{i}\right)<u_{i}\left(y^{\prime}, t_{i}^{\prime} ; v_{i}\right)$, which implies $(y, t) \notin I(v)$.

\section{A.4 Proof of Proposition 1}

Let $v \in V$. We proceed in two steps. ${ }^{12}$

Step 1: For each $\boldsymbol{i} \in\{\mathbf{1}, \mathbf{2}\}, \boldsymbol{U}\left(\boldsymbol{S}_{\boldsymbol{i}}^{\mathrm{VC}} ;\left(\boldsymbol{\Gamma}^{\mathrm{VC}}, \boldsymbol{v}\right)\right)=\left[\mathbf{0}, \boldsymbol{v}_{\boldsymbol{i}}[. \quad\right.$ Let $i \in\{1,2\}$. Without loss of generality, assume $i=1$.

\footnotetext{
${ }^{12}$ Let $a, b \in \mathbb{R}$ be such that $a \leq b$. Then, we denote by $[a, b]$ and $] a, b[$ the closed interval from $a$ to $b$ and the open interval from $a$ to $b$, respectively. We also denote by $[a, b[$ and $] a, b]$ the half-open intervals from $a$ to $b$.
} 
- Substep 1-1: Any $s_{1} \in\left[v_{1}, \omega_{1}\right]$ is weakly dominated. Let $s_{1} \in\left[0, v_{1}\left[\right.\right.$. Since $\omega_{2} \geq c$ by A1, there is $s_{2}^{*} \in S_{2}^{\mathrm{VC}}$ such that $s_{1}+s_{2}^{*}=c$. Let $s_{2}^{* *} \in S_{2}^{\mathrm{VC}}$ be such that $s_{1}^{\prime}+s_{2}^{* *}=c$. Note that $s_{2}^{* *}<s_{2}^{*}$. Let $s_{2}^{\prime} \in S_{2}^{\mathrm{VC}}$. There are three cases.

- Case 1: $\boldsymbol{s}_{\mathbf{2}}^{\prime} \in\left[\mathbf{0}, \boldsymbol{s}_{\mathbf{2}}^{* *}\left[\right.\right.$. Then, $g^{\mathrm{VC}}\left(s_{1}, s_{2}^{\prime}\right)=g^{\mathrm{VC}}\left(s_{1}^{\prime}, s_{2}^{\prime}\right)=(0,(0,0))$, which implies

$$
u_{1}\left(g_{1}^{\mathrm{VC}}\left(s_{2}, s_{2}^{\prime}\right) ; v_{1}\right)=\omega_{1}=u_{1}\left(g_{1}^{\mathrm{VC}}\left(s_{2}^{\prime}, s_{2}^{\prime}\right) ; v_{1}\right)
$$

- Case 2: $\boldsymbol{s}_{\mathbf{2}}^{\prime} \in\left[\boldsymbol{s}_{\mathbf{2}}^{* *}, \boldsymbol{s}_{\mathbf{2}}^{*}\left[\right.\right.$. Then, $g^{\mathrm{VC}}\left(s_{1}, s_{2}^{\prime}\right)=(0,(0,0))$ and $g^{\mathrm{VC}}\left(s_{1}^{\prime}, s_{2}^{\prime}\right)=\left(1,\left(-s_{1}^{\prime},-s_{2}^{\prime}\right)\right)$. Since $s_{1}^{\prime} \geq v_{1}$

$$
u_{1}\left(g_{1}^{\mathrm{VC}}\left(s_{1}, s_{2}^{\prime}\right) ; v_{1}\right)=\omega_{1} \geq v_{1}+\omega_{1}-s_{1}^{\prime}=u_{1}\left(g_{1}^{\mathrm{VC}}\left(s_{1}^{\prime}, s_{2}^{\prime}\right) ; v_{1}\right) .
$$

- Case 3: $\boldsymbol{s}_{\mathbf{2}}^{\prime} \in\left[\boldsymbol{s}_{2}^{*}, \boldsymbol{\omega}_{\mathbf{2}}\right]$. Then, $g^{\mathrm{VC}}\left(s_{1}, s_{2}^{\prime}\right)=\left(1,\left(-s_{1},-s_{2}^{\prime}\right)\right)$ and $g^{\mathrm{VC}}\left(s_{1}^{\prime}, s_{2}^{\prime}\right)=$ $\left(1,\left(-s_{1}^{\prime},-s_{2}^{\prime}\right)\right)$. Since $s_{1}^{\prime}>s_{1}$,

$$
u_{1}\left(g_{1}^{\mathrm{VC}}\left(s_{1}, s_{2}^{\prime}\right) ; v_{1}\right)=v_{1}+\omega_{1}-s_{1}>v_{1}+\omega_{1}-s_{1}^{\prime}=u_{1}\left(g_{1}^{\mathrm{VC}}\left(s_{1}^{\prime}, s_{2}^{\prime}\right) ; v_{1}\right) .
$$

By Cases 1-3, we can conclude that $s_{1}^{\prime}$ is weakly dominated.

- Substep 1-2: Any $s_{1} \in\left[0, v_{1}\right.$ [ is not weakly dominated. Note that there is $s_{2}^{*} \in S_{2}^{\mathrm{VC}}$ be such that $s_{1}+s_{2}^{*}=c$. Let $s_{1}^{\prime} \in S_{1}^{\mathrm{VC}} \backslash\left\{s_{1}\right\}$. There are two cases.

- Case 1: $\boldsymbol{s}_{\mathbf{1}}>\boldsymbol{s}_{\mathbf{1}}^{\prime}$. Then $g^{\mathrm{VC}}\left(s_{1}, s_{2}^{*}\right)=\left(1,\left(-s_{1},-s_{2}^{*}\right)\right)$ and $g^{\mathrm{VC}}\left(s_{1}^{\prime}, s_{2}^{*}\right)=(0,(0,0))$. Since $v_{1}>s_{1}$,

$$
u_{1}\left(g_{1}^{\mathrm{VC}}\left(s_{1}, s_{2}^{*}\right) ; v_{1}\right)=v_{1}+\omega_{1}-s_{1}>\omega_{1}=u_{1}\left(g_{1}^{\mathrm{VC}}\left(s_{1}^{\prime}, s_{2}^{*}\right) ; v_{1}\right) .
$$

- Case 2: $\boldsymbol{s}_{\mathbf{1}}<\boldsymbol{s}_{\mathbf{1}}^{\prime}$. Then, $g^{\mathrm{VC}}\left(s_{1}, s_{2}^{*}\right)=\left(1,\left(-s_{1},-s_{2}^{*}\right)\right)$ and $g^{\mathrm{VC}}\left(s_{1}^{\prime}, s_{2}^{*}\right)=\left(1,\left(-s_{1}^{\prime},-s_{2}^{*}\right)\right)$. Since $s_{1}^{\prime}>s_{1}$

$$
u_{1}\left(g_{1}^{\mathrm{VC}}\left(s_{1}, s_{2}^{*}\right) ; v_{1}\right)=v_{1}+\omega_{1}-s_{1}>v_{1}+\omega_{1}-s_{1}^{\prime}=u_{1}\left(g_{1}^{\mathrm{VC}}\left(s_{1}^{\prime}, s_{2}^{*}\right) ; v_{1}\right) .
$$

By Cases $1-2, s_{1}$ is not weakly dominated.

Step 2: $\mathcal{A}\left(\Gamma^{\mathrm{VC}}, \boldsymbol{v}\right) \subseteq \boldsymbol{C}(\boldsymbol{v})$. There are two cases.

- Case 1: $\boldsymbol{v}_{\mathbf{1}}+\boldsymbol{v}_{\mathbf{2}}>\boldsymbol{c}$. For each $i \in\{1,2\}$, let

$$
\underline{s}_{i} \equiv \inf \left\{s _ { i } \in \left[0, v_{i}\left[: s _ { i } + s _ { j } = c \text { for some } s _ { j } \in \left[0, v_{j}[\}\right.\right.\right.\right.
$$


Note that under A1, for each $s_{i} \in\left[0, \underline{s}_{i}\right]$ and each $s_{j} \in\left[0, v_{j}\left[, g^{\mathrm{VC}}\left(s_{i}, s_{j}\right)=(0,(0,0))\right.\right.$. ○ Substep 2-1: For each $i \in\{1,2\}$ and each $s_{i}^{\prime} \in\left[0, \underline{s}_{i}\right], s_{i}^{\prime}$ is weakly dominated in the game $\left(\Gamma^{\mathrm{VC}}(U), v\right)$. Let $i \in\{1,2\}$. Without loss of generality, assume $i=1$. Let $s_{1}, s_{1}^{\prime} \in\left[0, v_{1}\right.$ [ be such that $s_{1}^{\prime} \leq \underline{s}_{1}<s_{1}$. Then, there is $s_{2}^{*} \in\left[0, v_{2}\left[\right.\right.$ such that $s_{1}+s_{2}^{*}=c$. It follows that $g^{\mathrm{VC}}\left(s_{1}, s_{2}\right)=(0,(0,0))$ if $s_{2}<s_{2}^{*}$ and $g^{\mathrm{VC}}\left(s_{1}, s_{2}\right)=\left(1,\left(-s_{1},-s_{2}\right)\right)$ otherwise. Let $s_{2} \in\left[0, v_{2}[\right.$. We also distinguish two subcases.

1. If $s_{2}<s_{2}^{*}$, then $g_{1}^{\mathrm{VC}}\left(s_{1}, s_{2}\right)=g_{1}^{\mathrm{VC}}\left(s_{1}^{\prime}, s_{2}\right)=(0,(0,0))$, which implies

$$
u_{1}\left(g_{1}^{\mathrm{VC}}\left(s_{1}, s_{2}\right) ; v_{1}\right)=\omega_{1}=u_{1}\left(g_{1}^{\mathrm{VC}}\left(s_{1}^{\prime}, s_{2}\right) ; v_{1}\right) .
$$

2. If $s_{2} \geq s_{2}^{*}$, then $g_{1}^{\mathrm{VC}}\left(s_{1}, s_{2}\right)=\left(1,\left(-s_{1},-s_{2}\right)\right)$ and $g_{1}^{\mathrm{VC}}\left(s_{1}^{\prime}, s_{2}\right)=(0,(0,0))$. Since $v_{1}>s_{1}$,

$$
u_{1}\left(g_{1}^{\mathrm{VC}}\left(s_{1}, s_{2}\right) ; v_{1}\right)=v_{1}+\omega_{1}-s_{1}>\omega_{1}=u_{1}\left(g_{1}^{\mathrm{VC}}\left(s_{1}^{\prime}, s_{2}\right) ; v_{1}\right)
$$

Hence $s_{1}^{\prime}$ is weakly dominated.

- Substep 2-2: For each $i \in\{1,2\}$ and each $\left.s_{i} \in\right] \underline{s}_{i}, v_{i}\left[, s_{i}\right.$ is not weakly dominated in the game $\left(\Gamma^{\mathrm{VC}}(U), v\right)$. Let $i \in\{1,2\}$. Without loss of generality, assume $i=1$. Let $\left.s_{1} \in\right] \underline{s}_{1}, v_{1}\left[\right.$. Note that there is $s_{2}^{*} \in\left[0, v_{2}\left[\right.\right.$ such that $s_{1}+s_{2}^{*}=c$. Let $s_{1}^{\prime} \in\left[0, v_{1}\left[\backslash\left\{s_{1}\right\}\right.\right.$. We also distinguish two subcases.

1. If $s_{1}>s_{1}^{\prime}$, then $g_{1}^{\mathrm{VC}}\left(s_{1}, s_{2}^{*}\right)=\left(1,\left(-s_{1},-s_{2}^{*}\right)\right)$ and $g_{1}^{\mathrm{VC}}\left(s_{1}^{\prime}, s_{2}^{*}\right)=(0,(0,0))$. Since $v_{1}>s_{1}$,

$$
u_{1}\left(g_{1}^{\mathrm{VC}}\left(s_{1}, s_{2}^{*}\right) ; v_{1}\right)=v_{1}+\omega_{1}-s_{1}>\omega_{1}=u_{1}\left(g_{1}^{\mathrm{VC}}\left(s_{1}^{\prime}, s_{2}^{*}\right) ; v_{1}\right)
$$

2. If $s_{1}<s_{1}^{\prime}$, then $g_{1}^{\mathrm{VC}}\left(s_{1}, s_{2}^{*}\right)=\left(1,\left(-s_{1},-s_{2}^{*}\right)\right)$ and $g_{1}^{\mathrm{VC}}\left(s_{1}^{\prime}, s_{2}^{*}\right)=\left(1,\left(-s_{1}^{\prime},-s_{2}^{*}\right)\right)$. Since $s_{1}<s_{1}^{\prime}$,

$$
u_{1}\left(g_{1}^{\mathrm{VC}}\left(s_{1}, s_{2}^{*}\right) ; v_{1}\right)=v_{1}+\omega_{1}-s_{1}>v_{1}+\omega_{1}-s_{1}^{\prime}=u_{1}\left(g_{1}^{\mathrm{VC}}\left(s_{1}^{\prime}, s_{2}^{*}\right) ; v_{1}\right)
$$

Hence $s_{1}$ is not weakly dominated.

○ Substep 2-3: $\mathcal{E}\left(\Gamma^{\mathrm{VC}}, v\right)=\left\{\left(s_{1}, s_{2}\right) \in\left[0, v_{1}\left[\times\left[0, v_{2}\left[: s_{1}+s_{2}=c\right\}\right.\right.\right.\right.$. We first show that $\mathcal{E}\left(\Gamma^{\mathrm{VC}}, v\right) \subseteq\left\{\left(s_{1}, s_{2}\right) \in\left[0, v_{1}\left[\times\left[0, v_{2}\left[: s_{1}+s_{2}=c\right\}\right.\right.\right.\right.$. Let $s \in \mathcal{E}\left(\Gamma^{\mathrm{VC}}, v\right)$. Suppose, by contradiction, that $s_{1}+s_{2} \neq c$. If $s_{1}+s_{2}>c$, there is $i \in\{1,2\}$ with $s_{i}>0$. Let $\varepsilon>0$ 
be such that $s_{i}+s_{j}>c+\varepsilon$. Let $s_{i}^{\prime}=s_{i}-\varepsilon$. Then,

$$
u_{i}\left(g_{i}^{\mathrm{VC}}\left(s_{i}^{\prime}, s_{j}\right) ; v_{i}\right)=v_{i}+\omega_{i}-s_{i}+\varepsilon>v_{i}+\omega_{i}-s_{i}=u_{i}\left(g_{i}^{\mathrm{VC}}\left(s_{i}, s_{j}\right) ; v_{i}\right),
$$

a contradiction. If $s_{1}+s_{2}<c$, by Substeps 2.1 and 2.2, then $s_{1}>\underline{s}_{1}$ and $s_{2}>\underline{s}_{2}$. Let $i \in\{1,2\}$. Then, there is $s_{i}^{\prime}$ such that $s_{i}^{\prime}+s_{j}=c$. Since $v_{i}>s_{i}$,

$$
u_{i}\left(g_{i}^{\mathrm{VC}}\left(s_{i}^{\prime}, s_{j}\right) ; v_{i}\right)=v_{i}+\omega_{i}-s_{i}>\omega_{i}=u_{i}\left(g_{i}^{\mathrm{VC}}\left(s_{i}, s_{j}\right) ; v_{i}\right),
$$

a contradiction.

We next show that $\left\{\left(s_{1}, s_{2}\right) \in\left[0, v_{1}\left[\times\left[0, v_{2}\left[: s_{1}+s_{2}=c\right\} \subseteq \mathcal{E}\left(\Gamma^{\mathrm{VC}}, v\right)\right.\right.\right.\right.$. Let $s^{\prime}=\left(s_{1}^{\prime}, s_{2}^{\prime}\right) \in\left\{\left(s_{1}, s_{2}\right) \in\left[0, v_{1}\left[\times\left[0, v_{2}\left[: s_{1}+s_{2}=c\right\}\right.\right.\right.\right.$. Then, for each $i, j \in\{1,2\}$ and each $s_{i}^{\prime \prime}\left(>\underline{s}_{i}\right)$,

$$
u_{i}\left(g_{i}^{\mathrm{VC}}\left(s_{i}^{\prime}, s_{j}^{\prime}\right) ; v_{i}\right)=v_{i}+\omega_{i}-s_{i} \geq u_{i}\left(g_{i}^{\mathrm{VC}}\left(s_{i}^{\prime \prime}, s_{j}^{\prime}\right) ; v_{i}\right) .
$$

Moreover, since $s_{1}^{\prime}+s_{2}^{\prime}=c$, both $s_{1}^{\prime}$ and $s_{2}^{\prime}$ are not weakly dominated. Thus, $s^{\prime} \in$ $\mathcal{E}\left(\Gamma^{\mathrm{VC}}, v\right)$.

- Substep 2-4: Concluding. It follows from Substep 2-3 that $\mathcal{A}\left(\Gamma^{\mathrm{VC}}, v\right) \subseteq C(v)$.

- Case 2: $\boldsymbol{v}_{\mathbf{1}}+\boldsymbol{v}_{\mathbf{2}} \leq \boldsymbol{c}$. To show $\mathcal{A}\left(\Gamma^{\mathrm{VC}}, v\right) \subseteq C(v)$, we first show that $\mathcal{E}\left(\Gamma^{\mathrm{VC}}, v\right)=$ $\left[0, v_{1}\left[\times\left[0, v_{2}\left[\right.\right.\right.\right.$. It is obvious that $\mathcal{E}\left(\Gamma^{\mathrm{VC}}, v\right) \subseteq\left[0, v_{1}\left[\times\left[0, v_{2}[\right.\right.\right.$. Therefore, it suffices to show that $\left[0, v_{1}\left[\times\left[0, v_{2}\left[\subseteq \mathcal{E}\left(\Gamma^{\mathrm{VC}}, v\right)\right.\right.\right.\right.$. Let $\left(s_{1}, s_{2}\right) \in\left[0, v_{1}\left[\times\left[0, v_{2}\left[\right.\right.\right.\right.$. Since $s_{1}+s_{2}<c$, $g^{\mathrm{VC}}(s)=(0,(0,0))$. It follows that for each $i \in\{1,2\}, s_{i}$ is not weakly dominated in the game $\left(\Gamma^{\mathrm{VC}}(U), v\right)$ and, moreover, $\left(s_{1}, s_{2}\right)$ is a Nash equilibrium of the game $\left(\Gamma^{\mathrm{VC}}(U), v\right)$. Hence $\left(s_{1}, s_{2}\right) \in \mathcal{E}\left(\Gamma^{\mathrm{VC}}, v\right)$. It then follows that $\mathcal{A}\left(\Gamma^{\mathrm{VC}}, v\right)=\{(0,(0,0))\} \subseteq C(v)$.

\section{B Appendix: Implementation in iterated elimina- tion of weakly dominated strategies}

This section shows that the voluntary contribution mechanism cannot implement any sub-correspondence of the core in iterated elimination of weakly dominated strategies. To see this, suppose that $\omega_{1}=\omega_{2}=c=8$.

We now consider the case where $v=(7,7)$. Let $U_{i}^{1} \equiv U\left(S_{i}^{\mathrm{VC}} ;\left(\Gamma^{\mathrm{VC}}, v\right)\right)$ and $U^{1} \equiv$ $U_{1}^{1} \times U_{2}^{1}$. From the proof of Proposition 1 (Step 2), we obtain the following facts:

- For each $i \in\{1,2\}, U_{i}^{1}=[0,7[$; 
- For each $i \in\{1,2\}$ and each $s_{i} \in\left[0,1\left[, s_{i}\right.\right.$ is weakly dominated in the game $\left(\Gamma^{\mathrm{VC}}\left(U^{1}\right), v\right)$; and

- For each $i \in\{1,2\}$ and each $s_{i} \in U_{i}^{1} \backslash\left[0,1[=] 1,7\left[, s_{i}\right.\right.$ is not weakly dominated in the game $\left(\Gamma^{\mathrm{VC}}\left(U^{1}\right), v\right)$.

Therefore, for each $\left.i \in\{1,2\}, U_{1}^{2} \equiv U\left(U_{i}^{1} ;\left(\Gamma^{\mathrm{VC}}\left(U^{1}\right), v\right)\right)=\right] 1,7[$. We now show that for each $i \in\{1,2\}$ and each $s_{i} \in U_{i}^{2}, s_{i}$ is not weakly dominated in the game $\left(\Gamma^{\mathrm{VC}}\left(U^{2}\right), v\right)$. Let $i \in\{1,2\}$. Without loss of generality, assume $i=1$. Let $s_{1} \in U_{1}^{2}$. Note that there is $s_{2}^{*} \in U_{2}^{2}$ such that $s_{1}+s_{2}^{*}=c=8$. Let $s_{1}^{\prime} \in U_{1}^{2} \backslash\left\{s_{1}\right\}$. There are two cases.

- Case 1: $\boldsymbol{s}_{\mathbf{1}}>\boldsymbol{s}_{\mathbf{1}}^{\prime}$. Then, $g_{1}^{\mathrm{VC}}\left(s_{1}, s_{2}^{*}\right)=\left(1,\left(-s_{1},-s_{2}^{*}\right)\right)$ and $g_{1}^{\mathrm{VC}}\left(s_{1}^{\prime}, s_{2}^{*}\right)=(0,(0,0))$. Since $v_{1}=7>s_{1}$,

$$
u_{1}\left(g_{1}^{\mathrm{VC}}\left(s_{1}, s_{2}^{*}\right) ; v_{1}\right)=v_{1}+\omega_{1}-s_{1}=8+\left(7-s_{1}\right)>8=\omega_{1}=u_{1}\left(g_{1}^{\mathrm{VC}}\left(s_{1}^{\prime}, s_{2}^{*}\right) ; v_{1}\right)
$$

- Case 2: $s_{1}<s_{1}^{\prime}$. Then, $g_{1}^{\mathrm{VC}}\left(s_{1}, s_{2}^{*}\right)=\left(1,\left(-s_{1},-s_{2}^{*}\right)\right)$ and $g_{1}^{\mathrm{VC}}\left(s_{1}^{\prime}, s_{2}^{*}\right)=\left(1,\left(-s_{1}^{\prime},-s_{2}^{*}\right)\right)$. Since $s_{1}<s_{1}^{\prime}$,

$$
u_{1}\left(g_{1}^{\mathrm{VC}}\left(s_{1}, s_{2}^{*}\right) ; v_{1}\right)=v_{1}+\omega_{1}-s_{1}>v_{1}+\omega_{1}-s_{1}^{\prime}=u_{1}\left(g_{1}^{\mathrm{VC}}\left(s_{1}^{\prime}, s_{2}^{*}\right) ; v_{1}\right) .
$$

From Cases 1-2, we can conclude that $s_{1}$ is not weakly dominated. This fact implies that the set of strategies that survive iterated elimination of weakly dominated strategies is $] 1,7[\times] 1,7[$. We now consider the strategy profile $(2,2)$. Then, $(2,2) \in] 1,7[\times] 1,7\left[\right.$ but $g^{\mathrm{VC}}(2,2)=(0,(0,0)) \notin C(v)$. This implies that the voluntary contribution mechanism fails to implement any sub-correspondence of the core in iterated elimination of weakly dominated strategies.

\section{Appendix: Experimental instructions}

In this experiment, please remember that you cannot talk or communicate with other subjects. If there is communication, the experiment will be stopped at that point.

First, please confirm that the following items are on your desk. If any of the items are missing, please contact an experimenter.

- Instructions (this set of papers)

- Payoff table for practice 
- Record sheet for practice

- Pencil and eraser

\section{C.1 Overview}

In this experiment, at the beginning of each period, an experimenter will choose the person you are paired with from the other subjects at random. The person you are paired with will change each period. This experiment consists of 20 periods. As explained in more detail below, at the beginning of each period, you will make decisions using a computer assigned to you based on the "Payoff table". You will choose one integer number from 1 to 20 in each period. No subject knows who they have been paired with either during or after the experiment.

The rewards you receive after the experiment is complete are proportional to the total payoffs you earn throughout the 20 periods of the experiment. A detailed explanation of the rewards you receive will be provided later in Section C.4 (Rewards).

\section{C.2 Details}

You will choose one integer number from 1 to 20 in each period. Your payoff in each period is determined by the "Outcome" of the period. The outcome is determined by the number you choose as well as the number the other person chooses. Your "Payoff table" describes the relation between outcomes and the payoffs you earn.

Your "Payoff table" displays both your payoff and the other person's payoff when the number you choose and the number the other person chooses are determined. The following table is a part of the "Payoff table for practice".

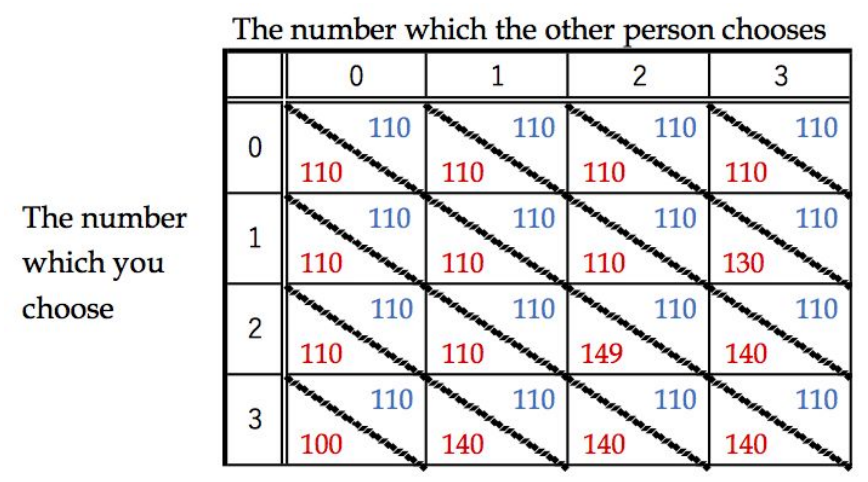

The vertical line of the above table shows the number you choose, and the horizontal line is the number the other person chooses. The lower left-hand red number is your 
payoff, and the upper right-hand blue number is the other person's payoff in each cell. The numbers in each cell are displayed in yen.

For example, suppose that the number you choose is " 3 ", and the number the other person chooses is " 1 ". In this case, your payoff is " 140 " when the vertical line is 3 and the horizontal line is 1 . Then, the other person's payoff is "100".

The person you are paired with is chosen from the other subjects at random by an experimenter. The person you are paired with has a payoff table in which the vertical line and the horizontal line of your payoff table are reversed.

\section{C.3 Operation}

This experiment consists of 20 periods. Here, we will explain how to operate the computer you will use in period 1 of the experiment. The operation after period 1 is the same as the operation in period 1.

1. At the beginning of period 1, you will be paired with the person an experimenter will choose from the other subjects at random. Then, "Input one integer number from 1 to 20" is displayed on the screen of your computer. The following figure is an example of the screen.

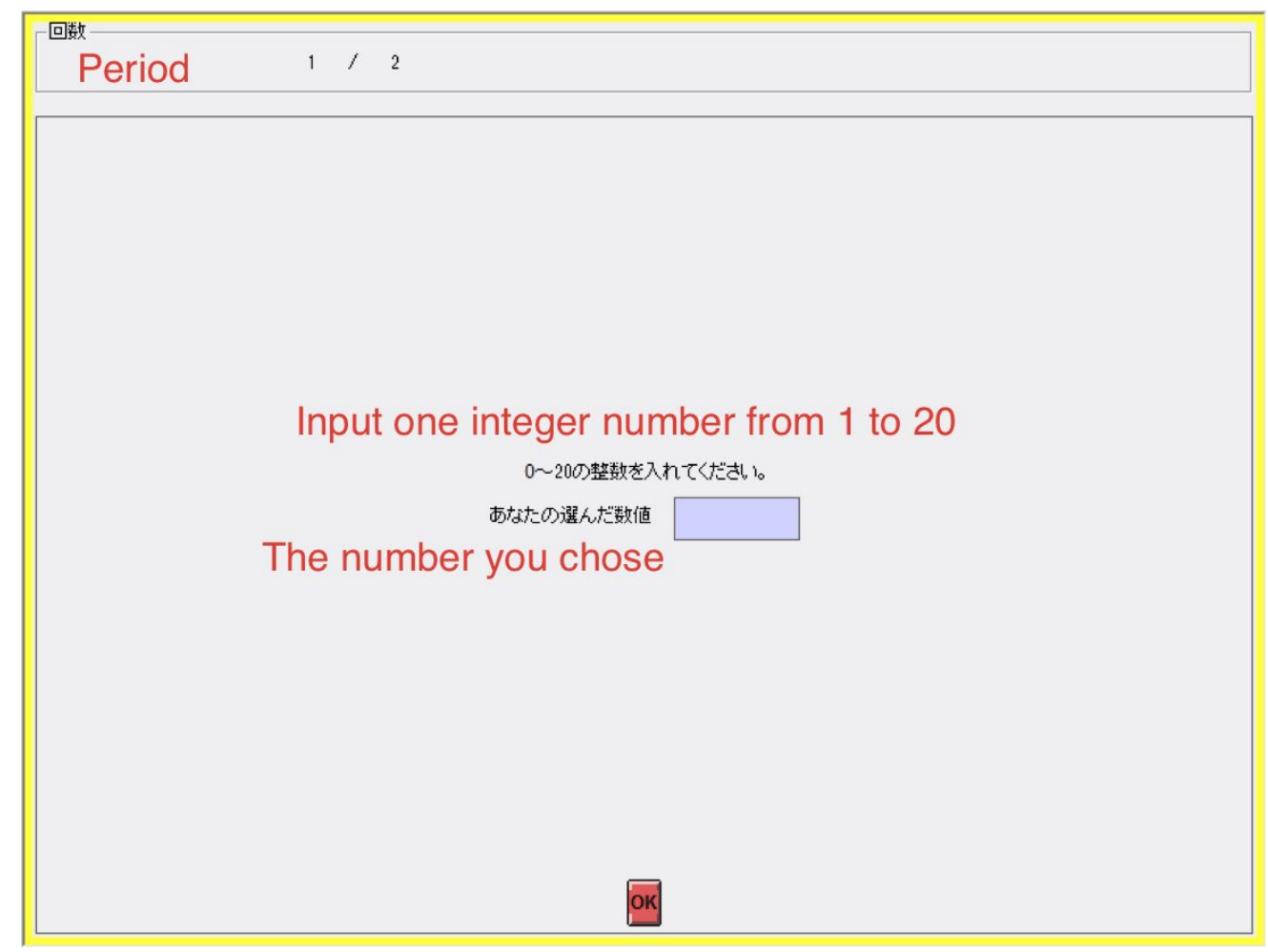


2. Please look at the "Payoff table" and confirm the relation between the numbers you and the other person choose and the payoffs.

3. After deciding which number to choose, you input that number in the cell of "The number you chose". Then, click the "OK" button at the bottom of the screen.

4. After inputting the number you chose on your computer, fill out the number you chose in the column "the number you chose" in the record sheet. Moreover, please fill out why you chose that number in the column "Reason for your decision" in the record sheet.

5. After all subjects click the "OK" button, period 1 is complete. Then, "The number you chose", "The number the other person chose", and "Your payoff" are displayed on the screen. We ask that you transcribe this information in the record sheet.

6. After the transcription, please the "NEXT" button at the bottom of the screen.

Once all subjects click the "NEXT" button, period 2 will start. At the beginning of period 2, you will be paired with the person an experimenter will choose from the other subjects at random. The operation after period 1 is the same as the operation in period 1 . The experiment is complete when period 20 is complete.

\section{C.4 Rewards}

Your rewards are the sum of your payoffs over all 20 periods. For example, if the sum of your payoffs are 2,580, then your rewards are 2,580 yen. If you earn more payoffs in each period, then you receive more rewards.

This concludes our explanation. Next, you can practice making decisions using the "Payoff table for practice". After practicing, you will have some time to look at the payoff table for the actual experiment before we begin the experiment. If you have any questions, please raise your hand. 


\section{References}

Attiyeh, G., R. Franciosi, and R. M. Isaac (2000) "Experiments with the pivotal process for providing public goods," Public Choice, 102, 95-114.

Bagnoli, M. and M. McKee (1991) "Voluntary contribution games: Efficient provision of public goods," Economic Inquiry, 29, 351-366.

Bagnoli, M. and B. L. Lipman (1989) "Provision of public goods: Fully implementing the core through private contributions," Review of Economic Studies, 56, 583-601.

Carbonell-Nicolau, O. (2011) "The existence of perfect equilibrium in discontinuous games," Games, 2, 235-256.

Cason, T., T. Saijo, T. Sjöström, and T. Yamato (2006) "Secure implementation experiments: Do strategy-proof mechanisms really work?," Games and Economic Behavior, 57, 206-235.

Chen, Y. (2008) "Incentive-compatible mechanisms for pure public goods: A survey of experimental research," in Handbook of Experimental Economics Results, Vol. 1, (V. Smith and C. Plott eds), North Holland, 625-643.

Chen, Y. and J. O. Ledyard (2010) "Mechanism design experiments," in Behavioral and Experimental Economics, (S. N. Durlauf and B. E. Lawrence eds), Springer, $191-205$.

Clarke, E. H. (1971) "Multipart pricing of public goods," Public Choice, 2, 19-33.

Fischbacher, U. (2007) "z-Tree: Zurich toolbox for readymade economic experiments," Experimental Economics, 10, 171-178.

Healy, P. J. (2006) "Learning dynamics for mechanism design: An experimental comparison of public goods mechanisms," Journal of Economic Theory, 129, 114-149

Kawagoe, T. and T. Mori (2001) "Can the pivotal mechanism induce truth-telling? An experimental study," Public Choice, 108, 331-354.

Marks, M. B. and R. T. A. Croson (1999) "The effect of incomplete information in a threshold public goods experiment," Public Choice, 99, 103-118.

Saijo, T., T. Sjöström, and T. Yamato (2007) "Secure implementation," Theoretical Economics, 2, 203-229. 